\title{
Gut epithelial TSC1/mTOR controls RIPK3-dependent necroptosis in intestinal inflammation and cancer
}

\author{
Yadong Xie, ${ }^{1,2}$ Yifan Zhao, ${ }^{1}$ Lei Shi, ${ }^{1}$ Wei Li, ${ }^{1}$ Kun Chen, ${ }^{2}$ Min Li, ${ }^{2}$ Xia Chen, ${ }^{1}$ Haiwei Zhang, ${ }^{3}$ Tiantian Li, ${ }^{1}$ \\ Yu Matsuzawa-Ishimoto, ${ }^{4}$ Xiaomin Yao, ${ }^{4}$ Dianhui Shao, ${ }^{1}$ Zunfu Ke, ${ }^{5}$ Jian Li, ${ }^{6}$ Yan Chen, ${ }^{3}$ Xiaoming Zhang, ${ }^{1}$ Jun Cui, ${ }^{7}$ \\ Shuzhong Cui, ${ }^{8}$ Qibin Leng, ${ }^{8}$ Ken Cadwell, ${ }^{4}$ Xiaoxia Li, ${ }^{9}$ Hong Wei, ${ }^{6}$ Haibing Zhang, ${ }^{3}$ Huabin Li, ${ }^{2,5}$ and Hui Xiao
}

\begin{abstract}
'The Center for Microbes, Development and Health, Chinese Academy of Sciences (CAS) Key Laboratory of Molecular Virology and Immunology, Institut Pasteur of Shanghai; CAS Center for Excellence in Molecular Cell Science; University of Chinese Academy of Sciences, CAS, Shanghai, China. ${ }^{2}$ Center for Allergic and Inflammatory Diseases \& Department of Otolaryngology, Head and Neck Surgery, Affiliated Eye, Ear, Nose and Throat Hospital, Fudan University, Shanghai, China. ${ }^{3}$ CAS Key Laboratory of Nutrition, Metabolism and Food Safety, Shanghai Institute of Nutrition and Health, University of Chinese Academy of Sciences, CAS, Shanghai, China. "Kimmel Center for Biology and Medicine, Skirball Institute of Biomolecular Medicine, New York University School of Medicine, New York, New York, USA. ${ }^{5}$ Department of Pathology, First Affiliated Hospital of Sun Yat-sen University, Guangzhou, Guangdong, China. ${ }^{6}$ Department of Laboratory Animal Science, College of Basic Medical Sciences, Third Military Medical University, Chongqing, China. 'Key Laboratory of Gene Engineering of the Ministry of Education, State Key Laboratory of Biocontrol, School of Life Sciences, Sun Yat-sen University, Guangzhou, Guangdong, China. ${ }^{8}$ State Key Laboratory of Respiratory Diseases, Affiliated Cancer Hospital and Institute of Guangzhou Medical University, Guangzhou Medical University, Guangzhou, Guangdong, China. ${ }^{9}$ Department of Inflammation and Immunity, Lerner Research Institute, Cleveland Clinic, Cleveland, Ohio, USA.
\end{abstract}

\begin{abstract}
Although Western diet and dysbiosis are the most prominent environmental factors associated with inflammatory bowel diseases (IBDs), the corresponding host factors and cellular mechanisms remain poorly defined. Here we report that the TSC1/mTOR pathway in the gut epithelium represents a metabolic and innate immune checkpoint for intestinal dysfunction and inflammation. mTOR hyperactivation triggered by Western diet or Tsc1 ablation led to epithelium necroptosis, barrier disruption, and predisposition to dextran sulfate sodium-induced colitis and inflammation-associated colon cancer. Mechanistically, our results uncovered a critical role for TSC1/mTOR in restraining the expression and activation of RIPK3 in the gut epithelium through TRIM11-mediated ubiquitination and autophagy-dependent degradation. Notably, microbiota depletion by antibiotics or gnotobiotics attenuated RIPK3 expression and activation, thereby alleviating epithelial necroptosis and colitis driven by mTOR hyperactivation. mTOR primarily impinged on RIPK3 to potentiate necroptosis induced by TNF and by microbial pathogen-associated molecular patterns (PAMPs), and hyperactive mTOR and aberrant necroptosis were intertwined in human IBDs. Together, our data reveal a previously unsuspected link between the Western diet, microbiota, and necroptosis and identify the mTOR/RIPK3/necroptosis axis as a driving force for intestinal inflammation and cancer.
\end{abstract}

\section{Introduction}

Inflammatory bowel diseases (IBDs), comprising Crohn's disease and ulcerative colitis, are chronic and relapsing inflammatory disorders manifested in the gastrointestinal (GI) tract $(1,2)$. Despite a continuous increase in IBD incidence worldwide, the complex molecular and cellular mechanisms underlying IBD pathogenesis remain incompletely understood, and the clinical treatments for IBDs are unsatisfactory (3-5). To date, metagenomic analysis on IBD patients has identified over 200 genetic risk loci involving a broad spectrum of physiological processes, namely innate immune recognition, adaptive immunity, autophagy, and ER stress $(2,6)$. Consistent with the importance of maintaining "microbial tolerance" in the gut, a diversity of innate receptor pathways, such as TLRs, Nod-like receptors, and dectins, have been linked to IBD pathogenesis (6). Moreover, the identification of ATG16L1 and $I R G M$ as IBD-susceptible genes has led to the discovery of auto-

Conflict of interest: The authors have declared that no conflict of interest exists. Copyright: @ 2020, American Society for Clinical Investigation.

Submitted: September 9, 2019; Accepted: January 14, 2020; Published: March 16, 2020.

Reference information: / Clin Invest. 2020;130(4):2111-2128.

https://doi.org/10.1172/JCl133264. phagy as an integral component of intestinal homeostasis and inflammation (7-9). Indeed, autophagy is involved in the secretion of antimicrobial peptides and resolution of ER stress in Paneth cells (7), as well as the control of inflammasome activation in enterocytes $(8,9)$. Nevertheless, genetic risk factors only account for a small fraction of IBD incidence (20\%-40\% for Crohn's disease and $10 \%$ for ulcerative colitis) (3). Recently, environmental factors - such as the Western diet, enriched with protein and fat, and microbiota dysregulation - have been considered as major contributors to the ever-increasing IBD incidence (2-5). Hence, it is widely believed that the etiology of IBD involves the complex interplay between host factors, microbiota, and dietary factors (2, 10). However, the underlying molecular and cellular components bridging these multilayered interactions remain largely elusive.

The intestinal epithelium comprises functionally distinct cell types such as absorptive enterocytes, secretory goblet cells, and Paneth cells, which together act as a physical barrier separating the microbiota in the lumen from the immune cells residing in the lamina propria $(4,5)$. Recently, the regulated necrotic cell death, namely necroptosis, has been implicated in the regulation of intestinal homeostasis (11-13). A number of proinflammatory and prodeath signals, such as TNF, IFNs, and pathogen-associated molecular 
patterns (PAMPs), are capable of triggering necroptosis, especially when caspase- 8 is inactivated $(13,14)$. The initiation of necroptosis involves the activation of protein kinases RIPK1 and RIPK3, which subsequently phosphorylate the executioner molecule MLKL to induce cell membrane rupture $(13,15-18)$. Often causing the release of a myriad of damage-associated molecular patterns, such as IL- $1 \alpha$, IL-1 $\beta$, and HMGB1, necroptosis has been regarded as a highly proinflammatory process that requires stringent control (13-15). Indeed, a number of negative regulators, such as caspase-8, FADD, A20, CHIP, c-FLIP, MK2, TBK1, and TAK1, have been implicated in the control of necroptosis (19-27). Remarkably, genetic ablation of either Casp 8 or Fadd in intestinal epithelial cells (IECs) is sufficient to induce necroptosis and spontaneous ileitis and/or colitis (19, 28). Moreover, aberrant RIPK3 activation and necrotic cell death have also been implicated in pediatric and adult IBDs $(19,29)$. Although the aforementioned studies indicate a causative role for RIPK3 and necroptosis in IBD, a protective role for RIPK3 in colitis has also emerged $(30,31)$. However, in the latter scenario, the antiinflammatory function of RIPK3 was restricted to the mononuclear phagocytes, which contrasts with its pronecroptotic function in the IECs (30-32). Therefore, the role of RIPK3 and necroptosis in IBD pathogenesis remains under debate $(33,34)$, and further studies are required to clarify its cell type-specific roles in intestinal homeostasis and inflammation.

mTOR plays crucial roles in cell growth/proliferation, development, immunity, and tumorigenesis in response to a diversity of extracellular and intracellular signals, including glucose, amino acids, cytokines, and PAMPs (35-38). Growth factors, cytokines, and TLR ligands trigger mTOR activation primarily through the phosphorylation and inactivation of the repressor TSC1/ TSC2 by a plethora of upstream protein kinases, such as PI3K/ AKT and ERK $(36,37,39)$. In contrast, nutrients such as amino acids induce mTOR activation mainly through the heteromeric GTPase Rags $(32,36)$. Once activated, mTOR functions through a myriad of downstream effectors to regulate diverse physiological processes, such as anabolism, autophagy, cell growth, and proliferation $(36,37)$. Under cell growth and rapid proliferation, mTOR is required to promote the phosphorylation of the translational regulator $4 \mathrm{E}-\mathrm{BP} 1$, as well as ribosome protein $\mathrm{S} 6$, both of which are vital regulators of protein synthesis. Moreover, under nutrient-sufficient conditions, mTOR can contribute to autophagy inhibition through phosphorylating and inactivating the autophagy initiator ULK1 (40). Further, the emerging evidence has also begun to link mTOR to intestinal homeostasis and tumorigenesis (41-45). Genetic ablation of mTOR in IECs revealed a critical role for mTOR in the regulation of caloric restriction (41), Paneth/goblet cell development $(42,44)$, and proliferation of transformed epithelium (43). Given the widespread association between mTOR dysregulation, chronic inflammation, and colon cancer, it becomes imperative to understand whether and how environmental factors might influence mTOR activation in the pathogenesis of IBD and colon cancer.

Breakdown of ingested nutrients, such as proteins, fatty acids, and carbohydrates in the GI tract, generates a variety of intermediary macromolecules, which can be further processed by microbiota $(4,10)$. Together, diet and microbiota constitute the most potent environmental factors profoundly shaping epithelium homeostasis and mucosal immunity $(5,46,47)$. As such, the Western diet and dysbiosis have been widely linked to IBD, although the corresponding host factors and cellular mechanisms are still poorly understood. Here we report that the epithelial TSC1/mTOR pathway is instrumental in the regulation of intestinal homeostasis, inflammation, and tumorigenesis. Our results further reveal a suprising role for mTOR in the regulation of RIPK 3 expression and necroptosis in the gut epithelium, a process profoundly shaped by the Western diet and microbiota. Overall, our findings support the emerging notion that epithelial necroptosis is a risk factor for intestinal inflammation and cancer, and provide new insight into the host response to dietary/microbial cues in the gut.

\section{Results}

The Western diet promotes mTOR hyperactivation and necroptosis in IECs. The Western diet is regarded as a major risk factor for IBD, yet how the Western diet, enriched with high protein and high fat, influences intestinal homeostasis and inflammation remains poorly understood. To this end, we fed mice with the Western diet containing either high protein (60\% protein) or high fat $(60 \%$ fat) for 2 weeks, followed by treatment with dextran sulfate sodium (DSS; MW $35-50 \mathrm{kDa}$ ) to induce intestinal inflammation. Compared with the mice fed a regular diet, high-protein- and high-fat-fed mice suffered more severe colonic inflammation, as demonstrated by the pronounced induction of proinflammatory genes (such as Tnf, Il1b, Il6, Cxcl1, Ccl2, and Ccl5), extensive ulceration, and heightened clinical symptoms in the colon in response to DSS treatment (Supplemental Figure 1, A-C; supplemental material available online with this article; https://doi.org/10.1172/JCI133264DS1). The mice fed with a high-protein or high-fat diet also displayed a high degree of morbidity and mortality (Supplemental Figure 1D and Figure 1, A and B). Moreover, prominent TUNEL-positive epithelial cells were detected in the colonic mucosae fed with a high-protein or high-fat diet (Figure 1C and Supplemental Figure 1E), hence associating IEC death with the Western diet. Given the recently emerged role for IEC necroptosis in intestinal inflammation (19, 27, 28), we wondered whether the necroptotic RIPK3/MLKL pathway could be involved in response to the Western diet. To test this scenario, we treated both WT and Mlkl-deficient mice with a high-protein diet for 2 weeks before DSS challenge. Although high-protein diet substantially exacerbated IEC death in WT mice, it failed to enhance IEC death or proinflammatory response in $M l k l$-deficient mice (Figure $1 \mathrm{C}$ and Supplemental Figure 1F), indicating a crucial role of MLKL in the Western diettriggered IEC death and colonic inflammation. By immunoblotting, we found that MLKL was markedly phosphorylated and activated in high-protein diet-treated IECs (Figure 1D). Similarly, RIPK3 phosphorylation was also highly induced in IECs pretreated with the high-protein diet (Figure 1D), hence correlating IEC necroptosis with the protein-enriched diet. Moreover, aberrant activation of the RIPK3/MLKL pathway was also associated with the IECs pretreated with a high-fat diet (Supplemental Figure 1G). These results hence reveal an unexpected link between the Western diet and IEC necroptosis in the pathogenesis of colitis.

Given the crucial role of mTOR in nutrient sensing, we sought to test the role of mTOR in Western diet-triggered epithelial 
A

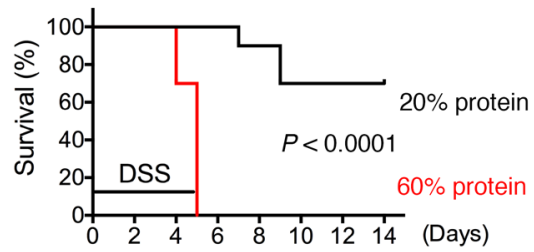

B

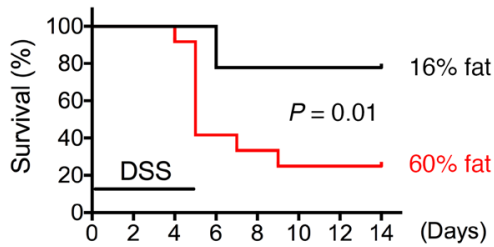

D

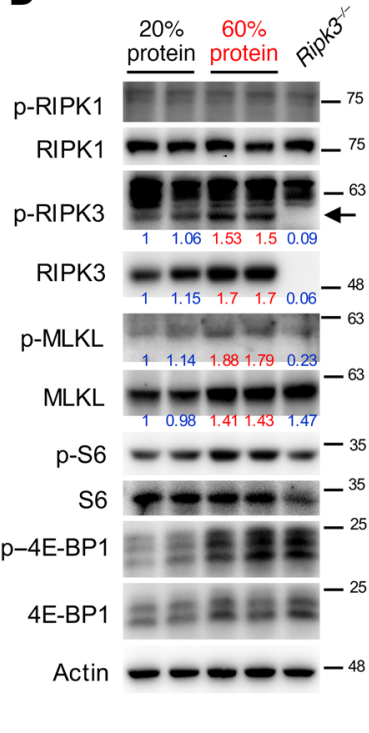

C

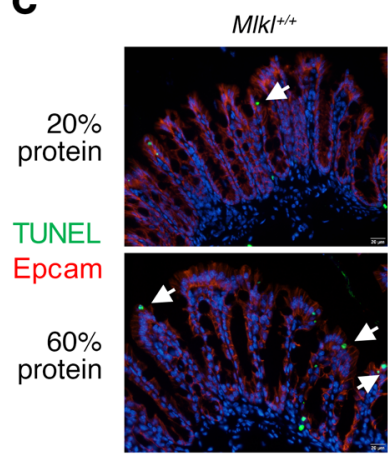

E

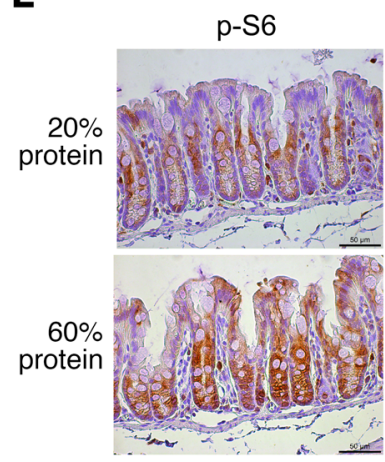

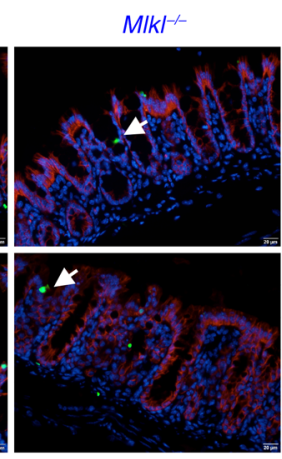

$\mathbf{F}$

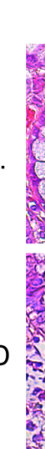

H\&E

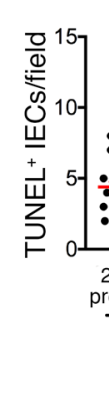

G

G TUNEL

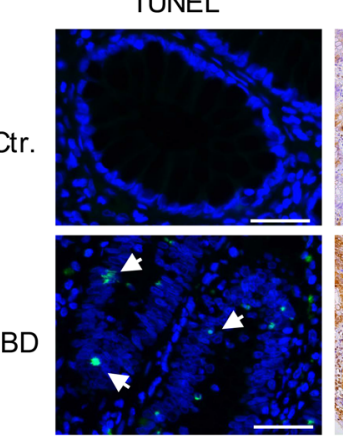

p-S6

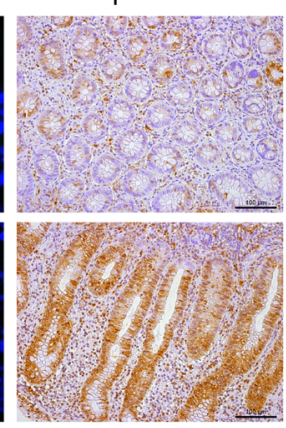

H

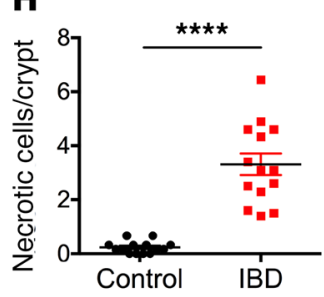

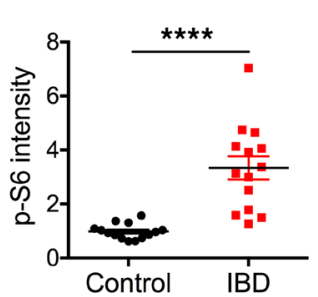

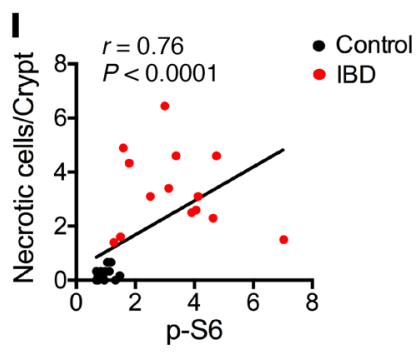

Figure 1. The Western diet promotes mTOR hyperactivation and necroptosis in the IECs. (A and B) C57BL/6 mice $(n=9-12)$ fed with a normal diet (20\% protein, $16 \%$ fat), high-protein diet (60\% protein), or high-fat diet (60\% fat) for 2 weeks were treated with $3.5 \%$ DSS in drinking water for 5 days, then switched to regular water. (C) Immunofluorescence (IF) staining of colon sections from WT and M/k/ $1^{-/-}$mice prefed with normal or high-protein diet and then challenged with $2.5 \%$ DSS for 5 days $(n=6)$. Epcam/TUNEL-double positive cells, denoted by arrows, were counted over 5 high-power fields $(x 400)$ per sample $(n=30)$. Scale bars: $20 \mu \mathrm{m}$. (D) Immunoblots of purified colonic epithelial lysates following $2.5 \%$ DSS treatment for 5 days on p-RIPK1 (S166), p-RIPK3 (S232), p-MLKL (S345), p-S6 (S235/236), or p-4EBP1 (Thr27/46). Numbers under Western blot bands represent relative quantifications over actin See complete unedited blots in the supplemental material. (E) IHC staining of colon sections fed with various protein diets for 2 weeks. Scale bars: $50 \mu \mathrm{m}$. (F and G) H\&E and immunostaining of control or IBD mucosal sections. Black arrows denote necrotic IECs with shrunken eosinophilic cytoplasm and pyknotic nuclei, and white arrows indicate TUNEL-positive IECs. Scale bars: $20 \mu \mathrm{m}$ (H\&E and TUNEL), $100 \mu \mathrm{m}$ (p-S6). (H) Statistical quantification of necrotic cell number and p-S6 staining intensity by Image (NIH) $(n=14)$. (I) Spearman's correlations between necrotic cell number and $p$-S6 intensity in control and IBD mucosae $(n=14)$. Data were pooled from 2 independent experiments (A and B) or are representative of 3 independent experiments $(\mathbf{C}-\mathbf{C})$ and are shown as mean \pm SEM. ${ }^{* * *} P<0.0001$, by log-rank test $(\mathbf{A}$ and $\mathbf{B})$ or unpaired Student's $t$ test $(\mathbf{C}$ and $\mathbf{H})$.

necroptosis. At first, we assessed the impact of the Western diet on epithelial mTOR activity by immunoblotting and immunohistochemical (IHC) staining. Following DSS challenge, S6 and $4 \mathrm{E}-\mathrm{BP} 1 / 2$, two well-known substrates of mTORC1, became greatly phosphorylated in the IECs pretreated with high-protein or highfat diet, indicative of mTOR activation by the Western diet (Figure 1D and Supplemental Figure 1G). Consistently, IHC revealed the presence of pronounced p-S6 in IECs treated with high-protein or 
high-fat diet as well (Figure 1E and Supplemental Figure 1H). Further, the Western diet induced prominent phosphorylation of AKT and GSK3 $\alpha / \beta$, two upstream kinases controlling mTOR activation through the TSC1/TSC2 complexes (refs. 48-50 and Supplemental Figure 1I). Consistently, the phosphorylation of TSC2 was also more abundantly present in the Western diet-treated IECs (Supplemental Figure 1I). These results revealed intestinal epithelial mTOR hyperactivation as a host response to the Western diet.

Based on the above observations, we sought to investigate the association between epithelial mTOR and necroptosis in human IBD, which has been strongly linked to the Western diet. We collected colon tissues from 14 patients with active Crohn's disease and examined mTOR activation via IHC staining. We also assessed IEC necroptosis in these samples by $\mathrm{H} \& \mathrm{E}$ and TUNEL staining. Histology analysis revealed the widespread presence of necrotic cells in the mucosa of IBD patients, especially in the inflamed area (Figure $1 \mathrm{~F}$ ). In contrast, epithelial cell death was barely detectable in the non-IBD controls (Figure 1F). Notably, the necrotic cells in the IBD mucosae displayed unique morphology and histology characteristics, such as disintegrated cell membrane, shrunken eosinophilic cytoplasm, and pyknotic nuclei (Figure 1F), thus resembling necroptotic cells as previously described $(19,29)$. TUNEL staining corroborated these results by demonstrating that there were higher numbers of necrotic cells in IBD mucosa than in non-IBD controls (Figure 1, G and H). By IHC analysis, we found that the majority of IBD samples exhibited a higher degree of p-S6 staining than control individuals (Figure 1, $\mathrm{G}$ and $\mathrm{H}$ ), and the abundance of $\mathrm{p}$-S6 was well correlated with the extent of necroptosis in IBD patients (Figure 1I). Together, these data indicate an interesting link between the Western diet, mTOR activation, and necroptosis in intestinal inflammation.

Epithelial TSC1/mTOR regulates necroptosis and intestinal barrier function. Next, we used a genetic approach to mimic the Western diet-triggered mTOR hyperactivation, specifically in the IEC compartment, to interrogate the role of epithelial mTOR in necroptosis and inflammation. By crossing Tsc1-floxed mice onto a Villin-cre transgenic strain, we generated a Tsc1-floxed Villin-cre $\left(T s c 1^{I E C-K O}\right)$ mouse strain in which the mTORC1 pathway was highly activated only in the IECs (Figure 2A). Unlike WT IECs, IECs isolated from $T s c 1^{I E C-K O}$ colonic crypts showed robust phosphorylation of RIPK3 and MLKL (Figure 2B), indicating the activation of the RIPK3/MLKL pathway. Consistently, the TUNEL assay revealed prominent epithelial cell death without caspase-3 activation in the $T s c 1^{I E C-K O}$ colon (Supplemental Figure 2A). These data suggest that TSC1/mTOR may be involved in the modulation of necroptosis in the IECs, possibly in cooperation with the well-defined necroptosis-eliciting signals such as TNF and/or microbialderived PAMPs $(19,28,51)$.

To further test this notion, we bred $\mathrm{Mlkl}^{-/-}$mice (52) onto the $T s c 1^{I E C-K O}$ strain, thereby generating $T s c 1^{I E C-K O} \mathrm{Mlkl}^{-/-}$mice, which presumably are refractory to necroptosis. As expected, the ablation of $M l k l$ alleviated the profound epithelial cell death in $T s c 1^{I E C-K O}$ colon, rendering Tsc1 ${ }^{I E C-K O} \mathrm{Mlkl}^{-/}$colon exhibiting a basal level of IEC death comparable to that seen in $\mathrm{Mlkl}^{l_{-}}$mice (Figure 2C). These data further support necroptosis as the major contributor to the aberrant IEC death in Tsc1 ${ }^{I E C-K O}$ mice. On the other hand, Ki-67 staining revealed mostly normal epithelial proliferation in the colon but slightly accelerated epithelial proliferation in the ileum of $T s c 1^{I E C-K O}$ mice (Supplemental Figure 2B). The histology analysis revealed largely normal presence of enterocytes and goblet cells in the $T s c 1^{I E C-K O}$ intestine (Supplemental Figure 2, C and D), except that fewer Paneth cells resided at the bottom of the ileum crypts (Supplemental Figure 2D). Additionally, the Tsc1 ${ }^{I E C-K O}$ ileum also expressed lower amounts of lysosome and defensin than WT ileum (Supplemental Figure 2E), further demonstrating a crucial role for TSC1/ mTOR in the maintenance and functionality of Paneth cells.

As intestinal barrier dysfunction has been frequently linked to the Western diet and aberrant epithelial cell death $(53,54)$, we went on to test whether mTOR hyperactivation might compromise epithelium barrier function. Following oral gavage, we found that about 2-fold more FITC-dextran (MW 3-5 kDa) was detected in the blood of $T s c 1^{I E C-K O}$ mice, indicative of disrupted epithelial barrier function (Figure 2D). Strikingly, the intestinal barrier dysfunction associated with TSC1 IEC deficiency was completely rescued upon the concomitant ablation of $M l k l$, suggesting a causative role for IEC necroptosis in intestinal barrier dysfunction (Figure 2D). Correspondingly, we found that a subset of proinflammatory genes, especially $I l 6, I l 1 b$, and $C x c l 1$, were upregulated in $T s c 1^{I E C-K O}$ colon (Figure $2 \mathrm{E}$ ). Moreover, a more significant number of immune cells, such as macrophages, neutrophils, and $\mathrm{T}$ cells, were infiltrated into the $T s c \mathrm{I}^{1 E C-K O}$ colon compared with WT controls (Figure 2F). These results collectively demonstrate that epithelial mTOR hyperactivation can lead to IEC necroptosis and homeostasis disruption in the gut.

Epithelial mTOR hyperactivation predisposes to severe colitis. Corresponding to the aforementioned disrupted homeostasis, $T s c 1^{I E C-K O}$ mice were highly susceptible to DSS-induced colitis. With a modest $2.5 \%$ of DSS challenge, Ts $C 1^{I E C-K O}$ mice exhibited extremely severe colitis, showing extensive ulceration, marked epithelial erosion, and pronounced infiltration of leukocytes (Supplemental Figure 3, A and B). Consistently, Tsc1 ${ }^{I E C-К O}$ mice displayed much worse clinical symptoms and considerably shortened colons compared with the WT controls (Supplemental Figure 3, C and D). Consequently, while marked morbidity and mortality were associated with $T s c 1^{I E C-K O}$ mice, all the WT mice survived from relatively mild colitis (Figure 3, A and B). These results collectively demonstrate that mTOR hyperactivation in the IECs can contribute to susceptibility to colitis.

Following DSS challenge, the intestinal barrier of $T s c 1^{I E C-K O}$ mice was further disrupted, leading to the presence of about 5-fold more FITC-dextran in their bloodstream than in that of WT mice (Figure 3C). Consistently, many proinflammatory genes, such as Tnf, Il1b, Il6, Cxcl1, Ccl2, and Ccl5, were highly expressed in the $T s c 1^{I E C-K O}$ colon following DSS treatment (Figure 3D), indicative of robust inflammatory response. Following DSS treatment, Tsc ${ }^{I E C-K O}$ IECs exhibited pronounced phosphorylation on RIPK3 and MLKL, along with elevated RIPK3 and MLKL expression as compared with the WT IECs (Figure 3E). However, the phosphorylation and expression levels of RIPK1 were comparable in WT and $T s c 1^{I E C-K O}$ IECs (Figure 3E). Moreover, while the genetic ablation of $M l k l$ abolished the expression and phosphorylation of MLKL, it had no impact on the activation of the upstream kinases RIPK1 and RIPK3 in both WT and Tsc1 ${ }^{I E C-K O}$ IECs (Figure 3E). Following DSS challenge, the prominent IEC death associated with 
A

Colon IEC

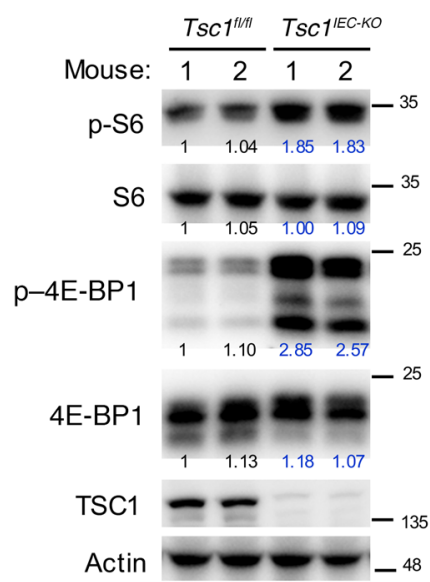

D

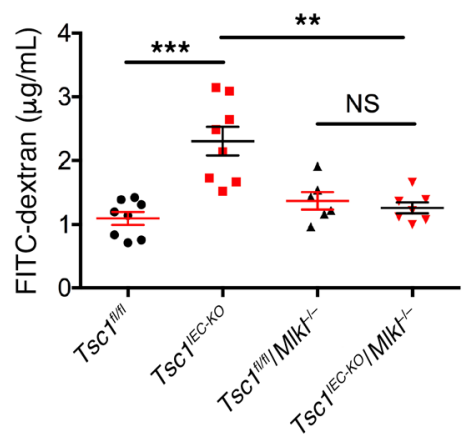

B

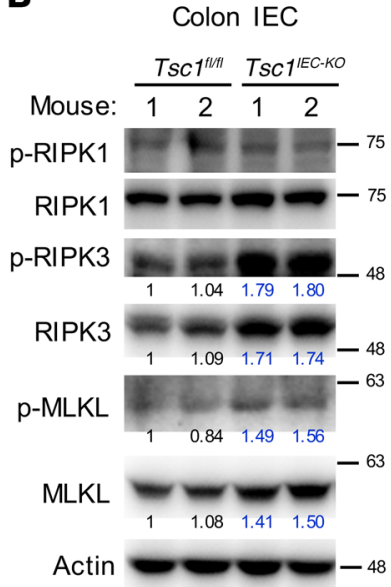

E

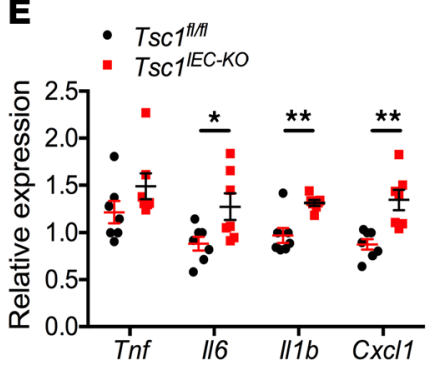

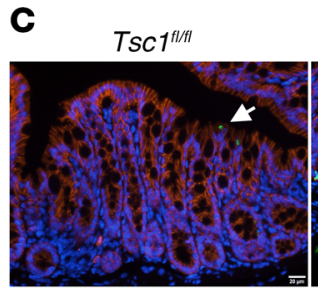

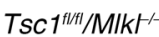
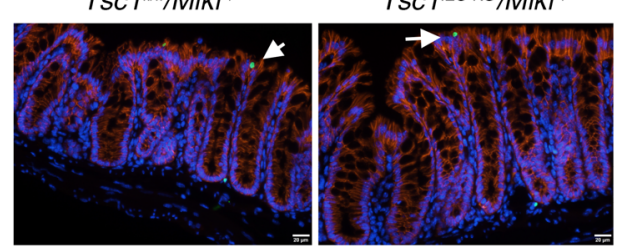

Epcam TUNEL

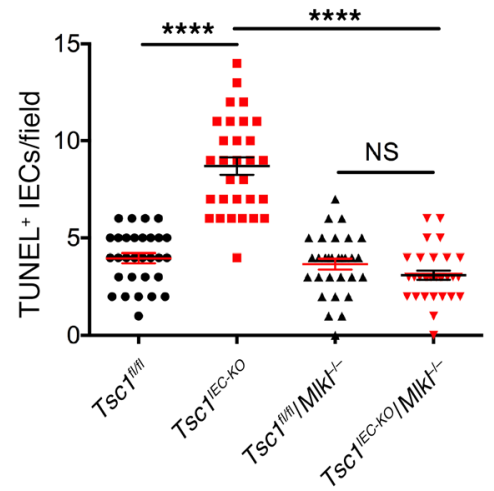

$\mathbf{F}$
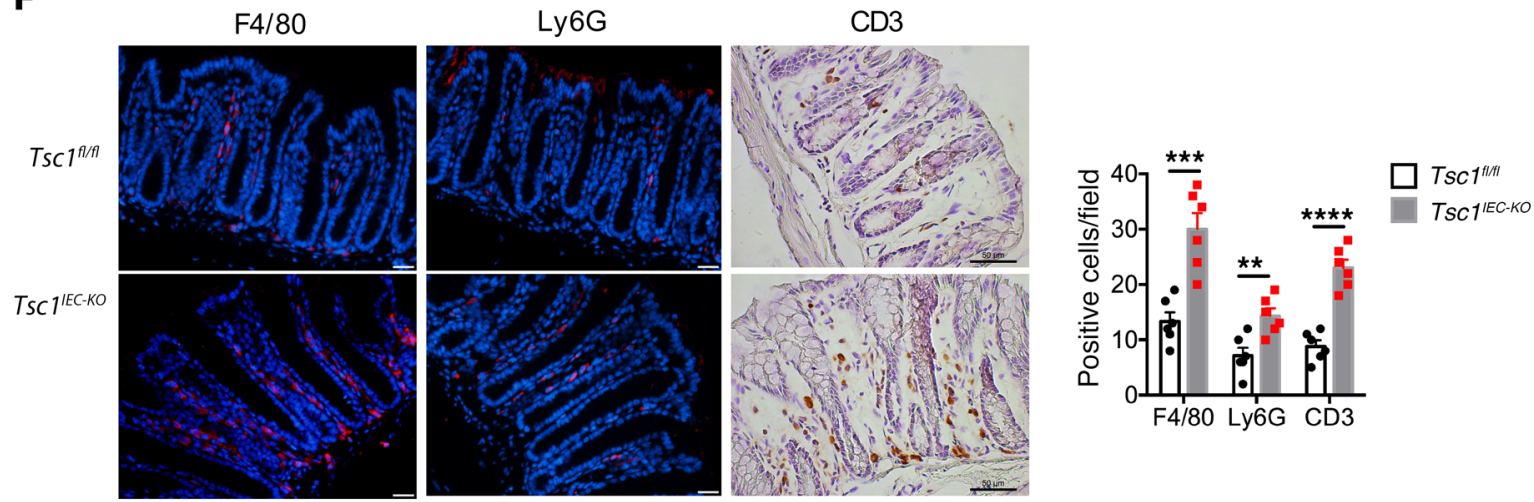

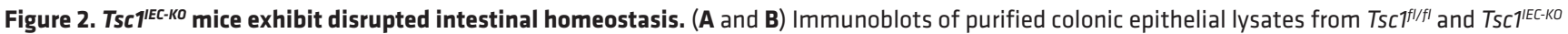
mice for p-RIPK1 (S166), p-RIPK3 (S232), p-MLKL (S345), p-S6 (S235/236), or p-4E-BP1 (Thr27/46). Numbers under Western blot bands represent relative quantifications over actin. (C) IF staining of colon sections $(n=6)$ from various genotypes. Epcam/TUNEL-double positive cells were counted over 5 high-power fields $(\times 400)$ per sample $(n=30)$. Scale bars: $20 \mu \mathrm{m}$. (D) Mice $(n=6-8)$ fasted for 4 hours were gavaged with FITC-dextran and bled in 4 hours. (E) Quantitative PCR (qPCR) analysis of inflammatory cytokine and chemokine mRNAs (normalized to $\beta$-actin) in steady-state colons ( $n=7$ ). Primers are listed in Supplemental Table 2. (F) IF staining and quantification of various immune cells in steady-state colons $(n=6)$. Scale bars: $20 \mu \mathrm{m}$ (F4/80 and Ly6C), $50 \mu \mathrm{m}$, (CD3). Data were pooled from 2 independent experiments ( $\mathbf{D}$ and $\mathbf{E}$ ) or are representative of 3 independent experiments $(\mathbf{A}-\mathbf{C}$ and $\mathbf{F})$ and are shown as mean \pm SEM. ${ }^{*} P<0.05,{ }^{* *} P<0.01,{ }^{* *} P<0.001,{ }^{* * *} P<0.0001$, by unpaired Student's $t$ test (E and $\left.\mathbf{F}\right)$ or 1 -way ANOVA (C and $\left.\mathbf{D}\right)$.

Tsc1 ${ }^{I E C-K O}$ colon was significantly alleviated upon the concomitant ablation of $M l k l$, and the $T s c I^{I E C-K O} M l k l^{-/}$compound mice showed a similar extent of IEC death to that in $\mathrm{Mlkl}^{\mathrm{H}^{-}}$mice (Figure $3 \mathrm{~F}$ ). Consistently, both $\mathrm{TsCI}^{\mathrm{IEC-KO}} \mathrm{Mlk \textrm {l } ^ { - - }}$ and $\mathrm{Mlkl}^{\mathrm{F}^{--}}$mice also had comparable colitis symptoms induced by DSS (Figure 3G), implicating an indispensable role for MLKL in driving the severe colitis in
$T s c 1^{I E C-K O}$ mice. Together, these data strongly indicate that epithelial necroptosis executed by MLKL has a crucial role in promoting intestinal dysfunction and overt inflammation in $T s c 1^{I E C-K O}$ mice.

TSC1 controls the microbiota/mTOR axis in IEC necroptosis and colitis. Given the above association of MTOR hyperactivation with epithelial necroptosis, we then rigorously interrogated the role of 
A

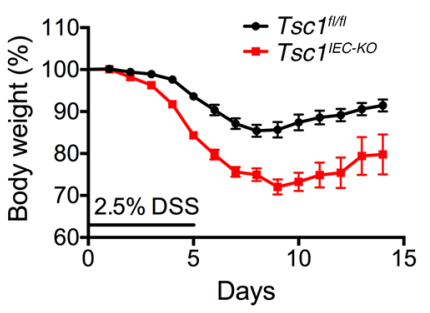

B

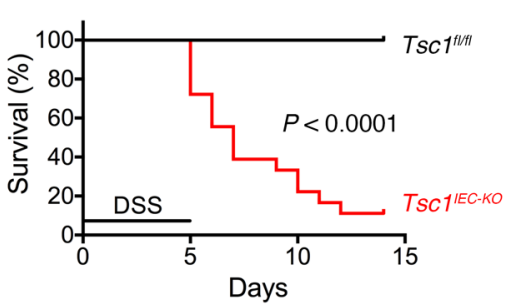

C

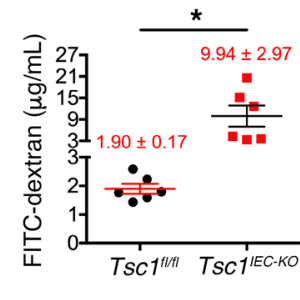

D

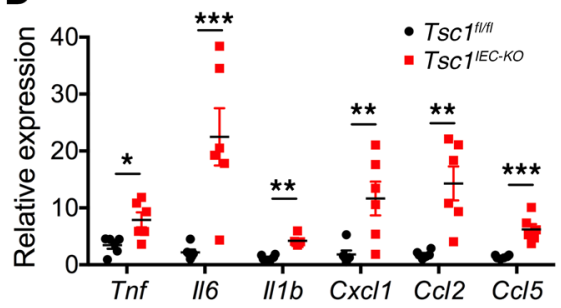

$\mathbf{F}$

$\mathbf{F}-T_{S C 1} 1^{t / 1 / t}$

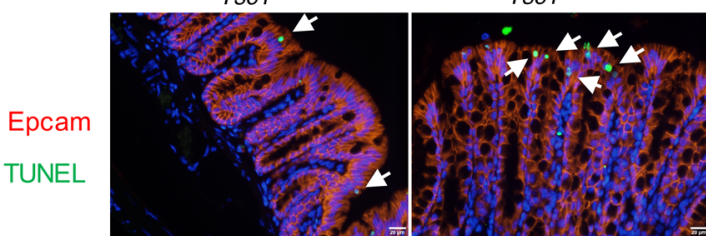

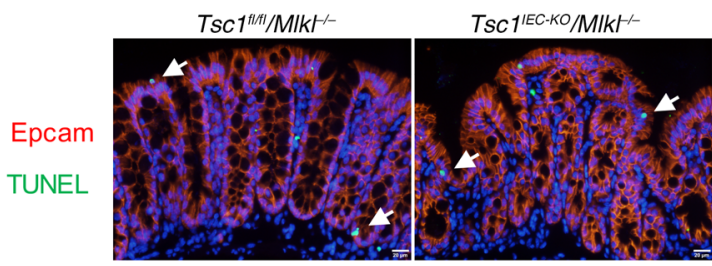

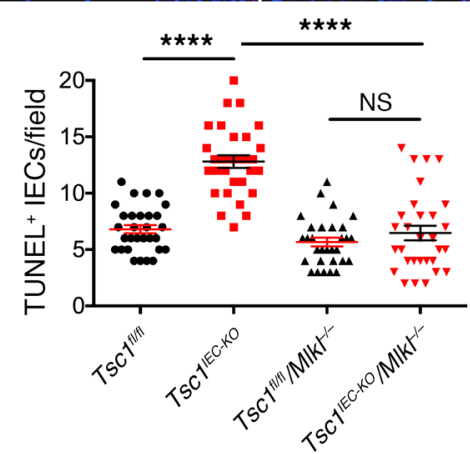

E

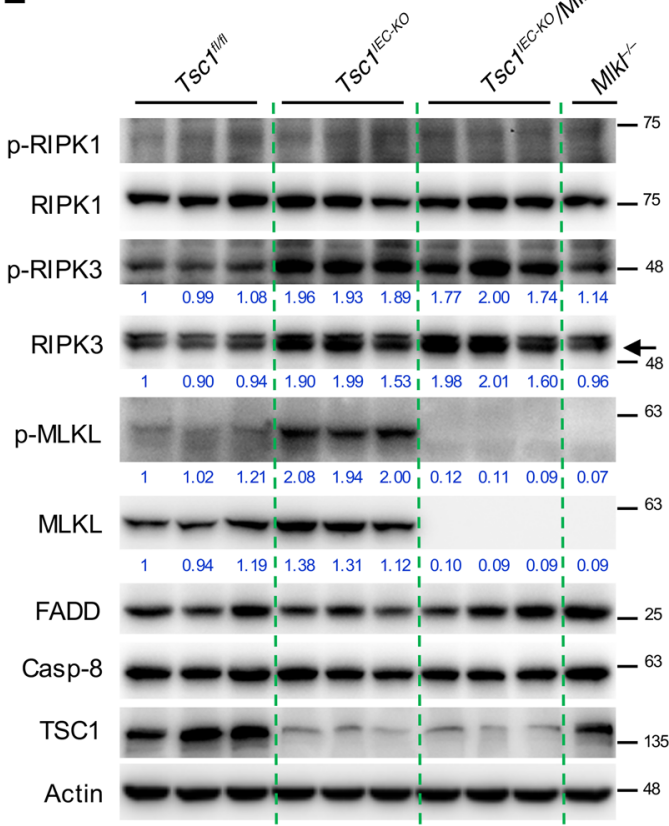

G

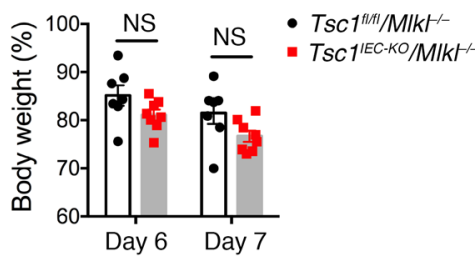

Figure 3. Aberrant epithelial necroptosis contributes to heightened colitis in Tsc ${ }^{1 E C-K O}$ mice. TSC ${ }^{f / / f l}$ and $T S C 7^{1 E C-K O}$ mice were treated with $2.5 \%$ DSS for 5 days and then with regular water. (A and B) Body weight (A) and survival (B) of the treated mice $(n=16-18)$ were documented daily. (C) Leakage of FITC-dextran from the Gl tract to the bloodstream was measured in DSS-treated mice for 3 days $(n=6)$. (D) qPCR analysis of proinflammatory gene expression (normalized to $\beta$-actin) in colons treated with DSS for 5 days $(n=6)$. (E) Immunoblots of colonic epithelial lysates from mice treated with DSS for 5 days $(n=3)$. p-RIPK1 (S166), p-RIPK3 (S232), and p-MLKL (S345) antibodies were used. Numbers under Western blot bands represent relative quantifications over actin. (F) IF staining on colon sections from various genotypes of mice treated with DSS for 5 days $(n=6)$. Double-positive cells were counted over 5 high-power fields $(\times 400)$ per sample $(n=30)$. Scale bars: $20 \mu \mathrm{m}$. (G) Body weight of mice treated with DSS for 5 days $(n=7-8)$. Data were pooled from 3 independent experiments (A, B, and $\mathbf{G})$ or are representative of 3 independent experiments $(\mathbf{C}-\mathbf{F})$ and are shown as mean \pm SEM. ${ }^{*} P<0.05,{ }^{* *} P<$ $0.01,{ }^{* *} P<0.001,{ }^{* * *} P<0.0001$, by log-rank test $(\mathbf{B})$, unpaired Student's $t$ test $(\mathbf{C}, \mathbf{D}$, and $\mathbf{C})$, or 1-way ANOVA (F).

mTOR in IEC death and gut inflammation. At first, we attempted to alleviate mTOR activation in WT and $T s c 1^{-/-}$IECs by intraperitoneally administering rapamycin. Following consecutive intraperitoneal injections of rapamycin for 5 days, TUNEL-positive epithelial cells were markedly reduced in $T s c 1^{I E C-K O}$ colon, but unaltered in WT colon (Figure 4A). Notably, rapamycin treatment largely reversed the aberrant IEC death in TSC $1^{I E C-K O}$ colon, leading to restored homeostasis as observed in WT colon (Figure 4A). Upon $2.5 \%$ DSS challenge, rapamycin-pretreated $T s c 1^{I E C-K O}$ mice showed a notable reduction in colitis symptoms, including inflammation, 
histopathology, morbidity, and mortality, compared with vehicletreated $T s c 1^{I E C-K O}$ mice (Supplemental Figure 4, A and B, and Figure 4 , B and C). On the contrary, the rapamycin pretreatment regimen used here (rapamycin treatment for 3 days before DSS challenge and 2 days after DSS challenge) failed to ameliorate colitis symptoms in WT mice (Supplemental Figure 4, A and B, and Figure 4, $B$ and $C$ ). Notably, more extended rapamycin treatment started after the DSS challenge has been associated with either reduced or enhanced colitis in WT mice $(55,56)$. Hence, it seems possible to ameliorate IEC death and colitis by tempering mTOR activity. Subsequently, we sought to alleviate mTOR activity in $T s c 1^{I E C-K O}$ IECs through a genetic approach. In an attempt to generate IEC-specific TSC1/mTOR-double knockout mice, we failed to obtain Tsc $1^{f / f l} / m T_{\text {Tor }}{ }^{l / f l}$ Villin-cre mice because of their embryonic lethality. Nevertheless, we found that $T s c 1^{f l / f l} / m T_{0} r^{f /+}$ Villin-cre mice (herein referred to as Tsc ${ }^{I E C-K O} / m$ Tor $^{I E C-H e t}$ ), with only $1 m$ Tor allele deleted in the Tsc ${ }^{I E C-K O}$ IECs, showed considerable reduction in mTOR activity (Figure 4D). Strikingly, assessment of necroptotic signaling by immunoblotting revealed diminished expression and phosphorylation of RIPK3 in Tsc $1^{I E C-K O} / m_{\text {Tor }}^{\text {IEC-Het }}$ IECs (Figure $4 \mathrm{D})$. Further, following DSS challenge, $T s c 1^{I E C-K O} / m$ Tor $^{I E C-H e t}$ mice suffered a lower degree of tissue damage (Supplemental Figure $4 \mathrm{C}$ ) and survived much better than $T s c 1^{I E C-K O}$ mice (Figure $4 \mathrm{E}$ ). These data establish a central role for the TSC1/mTOR axis in the control of intestinal homeostasis and inflammation.

Besides dietary nutrients, the TSC1/mTOR pathway has also been implicated in the interaction with microbiota (57), another environmental factor strongly tied to IBD. Interestingly, microbialderived PAMPs such as LPS, poly(I:C), and flagellin were able to promote mTOR activation in the ex vivo-cultured enteroid cells (Supplemental Figure 4D). To determine the influence of microbiota on epithelial mTOR and necroptosis in vivo, we implemented both germ-free and antibiotics-depletion models. Strikingly, while the tonic phosphorylation of RIPK3/MLKL was evidently observed in IECs isolated from mice maintained in a specific pathogen-free facility, RIPK3/MLKL activation became barely detectable in germ-free IECs (Figure 4F). It is noteworthy that the expression levels of RIPK3 and MLKL proteins also diminished in germ-free IECs (Figure 4F). Remarkably, the phosphorylation of $4 \mathrm{E}-\mathrm{BP} 1 / 2$ was considerably lower in germ-free IECs, indicative of decreased mTOR activity (Figure $4 \mathrm{~F}$ ). These data suggest that the activation of both RIPK3/MLKL and MTOR in the IECs is contingent on the microbiota.

Next, we depleted microbiota by orally administering a cocktail of antibiotics in drinking water and then examined necroptotic signaling in 5 weeks. Notably, microbial depletion through antibiotics alleviated RIPK3 protein accumulation and RIPK3 phosphorylation in Tsc1 ${ }^{I E C-K O}$ IECs (Supplemental Figure 4E). Moreover, antibiotics treatment also dampened the phosphorylation of MLKL in IECs, further supporting a crucial role for the microbiota in the activation of necroptotic signaling (Supplemental Figure 4E). Following DSS treatment, antibiotics-treated $T s c 1^{I E C-K O}$ mice had very mild inflammation and tissue damage (Supplemental Figure 4, F and $\mathrm{G}$ ), showing significantly improved clinical manifestation and morbidity as compared with the antibiotics-untreated Tsc1 ${ }^{I E C-K O}$ mice (Figure 4G and Supplemental Figure 4H). Antibiotics treatment also alleviated DSS-induced colitis in WT mice (Figure 4G and Supplemental Figure 4, F-H). Hence, microbiota and mTOR may form a liaison in regulating epithelial necroptosis and colitis.

RIPK3 overexpression underlies mTOR-promoted necroptosis and colitis. The studies shown above revealed that MTOR hyperactivation, associated with either the Western diet (Figure 1D and Supplemental Figure 1G) or Tsc1 ablation (Figure 2B, Figure 3E, and Figure 4D), was able to promote RIPK3 activation without influencing RIPK1, hence ruling out a possible role for mTOR in the upstream components of TNF- and TLR3-elicited necroptotic signaling, such as TAK1, TBK1, or Trif. It is also noteworthy that in addition to its phosphorylation, the expression of RIPK3 was also substantially increased upon mTOR hyperactivation (Figure 1D, Figure 2B, Figure 3E, Figure 4D, and Supplemental Figure 1G). Based on these observations, we reasoned that RIPK3 overexpression might underlie mTORpromoted necroptosis and colitis. To rigorously test this hypothesis, we first validated the quality of purified IECs in this study. By comparing the expression of Epcam in purified IECs versus the whole colon tissue, we observed profound enrichment of the epithelium marker Epcam in purified colonic epithelial cells, thus validating our IEC purification approach (Figure 5A). Notably, the increase in RIPK3 expression was more robust in $T s c 1^{I E C-K O}$ IECs than in the whole colon tissue of Tsc $1^{I E C-K O}$ mice (Figure 5A), highlighting the IEC-specific RIPK3 accumulation in response to $\mathrm{mTOR}$ hyperactivation.

To definitively address whether RIPK3 overexpression per se contributes to IEC necroptosis, we fine-tuned RIPK3 expression in the IECs through a genetic approach. As RIPK3 can exert a protective effect on colitis through a necroptosis-independent mechanism $(30,31)$, to avoid the confounding effect from RIPK3 deficiency, we took advantage of Ripk3 heterozygosity, which does not exhibit notable phenotype on the WT background (30). Strikingly, ablating only 1 allele of the Ripk3 gene in the $T s c 1^{I E C-K O}$ mice considerably alleviated epithelium RIPK3 expression, rendering $T s c 1^{I E C-K O} /$ Ripk $^{+/-}$IECs expressing similar levels of RIPK3 proteins to WT and Ripk3 ${ }^{+/-}$IECs (Figure 5B). Consistent with attenuated RIPK3 expression, the phosphorylation and activation of RIPK3 (Figure 5B), as well as epithelial cell death, were markedly reduced in $T s c 1^{I E C-K O} /$ Ripk3 $^{+/-}$IECs as compared with $T s c 1^{I E C-K O}$ IECs (Figure $5 C)$. These results collectively implicate RIPK3 overexpression as the driving force for aberrant necroptosis in the Tsc ${ }^{I E C-K O}$ IECs. In support of this notion, Ripk3 heterozygosity restored the intestinal barrier function in Tsc1 ${ }^{E \mathrm{EC}-\mathrm{KO}}$ mice, as $T s c 1^{I E C-K O} / \mathrm{Ripk}^{+/-}$and Ripk3 $^{+/-}$mice exhibited similar levels of FITC-dextran in their blood circulation (Figure 5D). Following DSS challenge, Tsc1 ${ }^{1 E C-K O} /$ $R i p k 3^{+/-}$mice had attenuated inflammation/tissue damage in the colon (Supplemental Figure 5, A-C) as well as modest weight loss/ morbidity compared with $T s c 1^{I E C-K O}$ mice (Figure 5E). Interestingly, Ripk3 $^{+/-}$mice showed similar colitis symptoms (Figure 5E and Supplemental Figure 5, A-C) and IEC death (Supplemental Figure 5D) to those of the WT mice. Overall, these data highlight the necessity for precision control of RIPK3 expression in the IECs.

mTOR regulates ubiquitin-dependent RIPK3 degradation via autophagy. Considering that excessive RIPK3 can impose such a detrimental effect, we went on to investigate how mTOR impacts RIPK3 abundance. Although RIPK3 overexpression exhibited in $T s c 1^{I E C-K O}$ IECs resembles what has been shown in $\operatorname{Casp} 8^{I E C-K O}$ and 
A
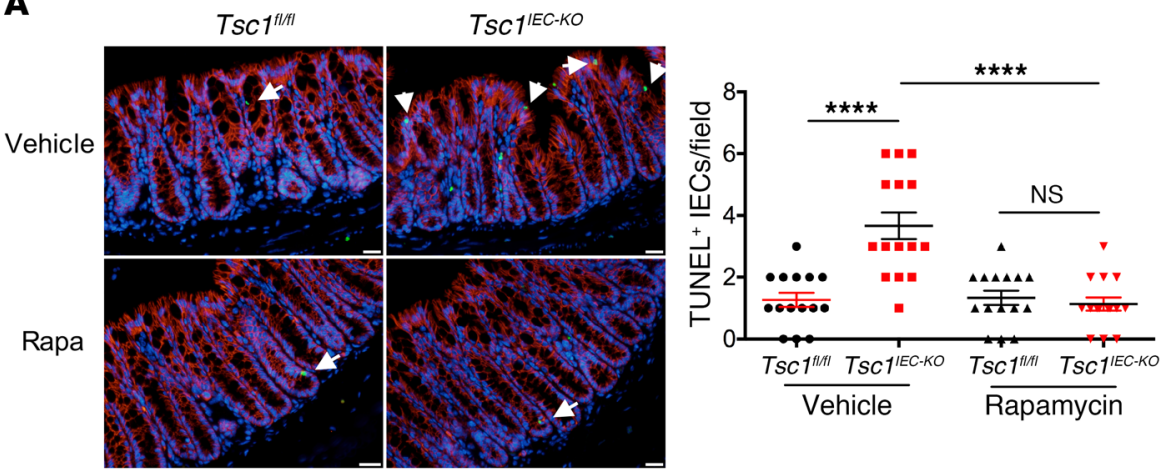

B

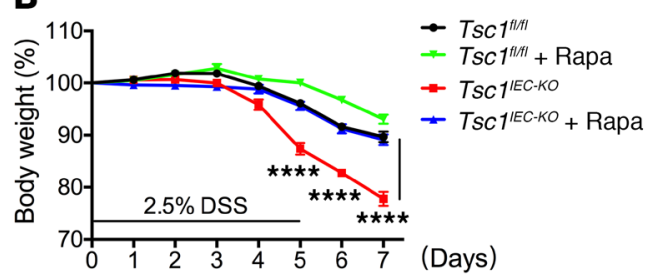

D

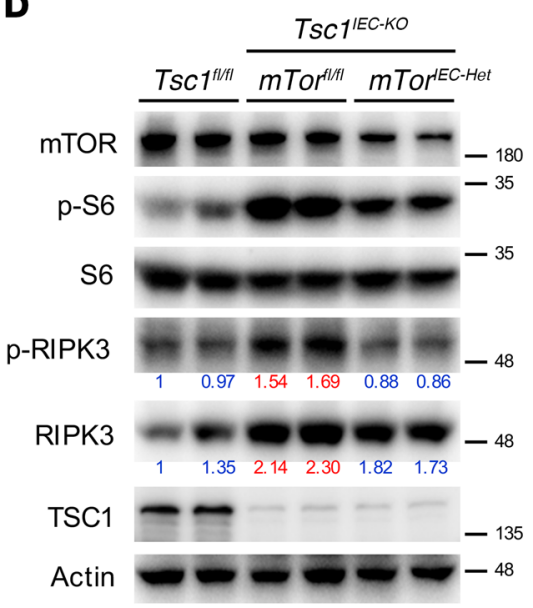

C

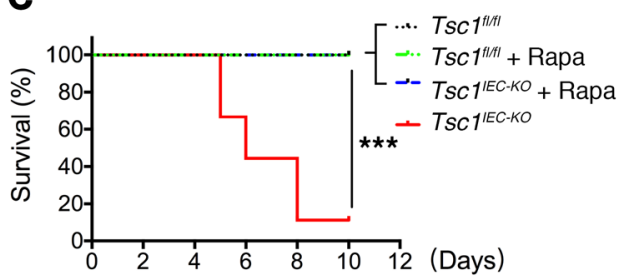

$\mathbf{F}$

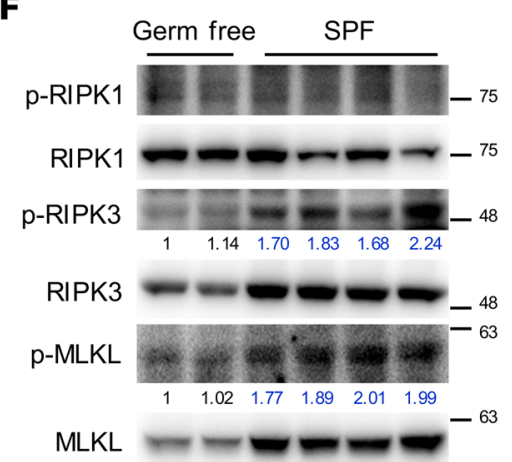

p-4E-BP1 $\quad \mathrm{OP}=-{ }^{25}$

$4 \mathrm{E}-\mathrm{BP} 1$

Actin

G

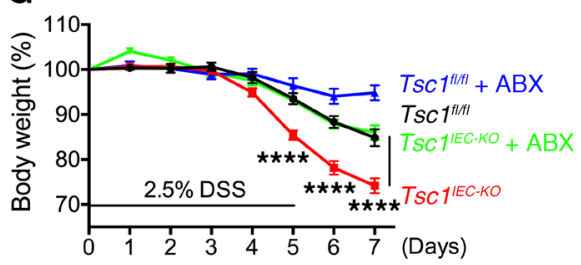

Figure 4. The microbiota/mTOR axis regulates epithelial necrosis and colitis. (A) Mice were intraperitoneally injected with vehicle or rapamycin ( $5 \mathrm{mg} / \mathrm{kg}$ for 5 days), colon sections $(n=5)$ were stained by IF, and double-positive cells were quantified over 15 images. Scale bars: $20 \mu \mathrm{m}$. (B and C) Three days after rapamycin treatment, mice were treated with $2.5 \%$ DSS for 5 days in the presence of rapamycin for only 2 days, and body weight (B) and survival (C) were recorded daily. (D) Immunoblots of colonic epithelial lysates from the indicated mice $(n=2)$. (E) Survival curve of mice treated with $2.5 \%$ DSS for 5 days $(n=12)$. (F) Immunoblots of colonic epithelial cell lysates from B6 mice maintained in a germ-free or specific pathogen-free facility ( $n=2-4)$. p-RIPK1 (S166), p-RIPK3 (S232), p-MLKL (S345), p-S6 (S235/236), and p-4E-BP1 (Thr27/46) were used. Numbers under Western blot bands represent relative quantifications over actin. (C) Mice untreated or pretreated with antibiotics (ABX) for 5 weeks were challenged with $2.5 \%$ DSS for 5 days to induce colitis, and body weights were recorded daily $(n=5)$. Data were pooled from 3 independent experiments $(\mathbf{B}, \mathbf{C}$, and $\mathbf{E})$ or are representative of 3 independent experiments $(\mathbf{A}, \mathbf{D}, \mathbf{F}$, and G) and are shown as mean \pm SEM. ${ }^{* *} P<0.001,{ }^{* * *} P<0.0001$, by log-rank test (C and $\left.\mathbf{E}\right)$, unpaired Student's $t$ test (B and $\left.\mathbf{G}\right)$, or 1-way ANOVA (A). 
A

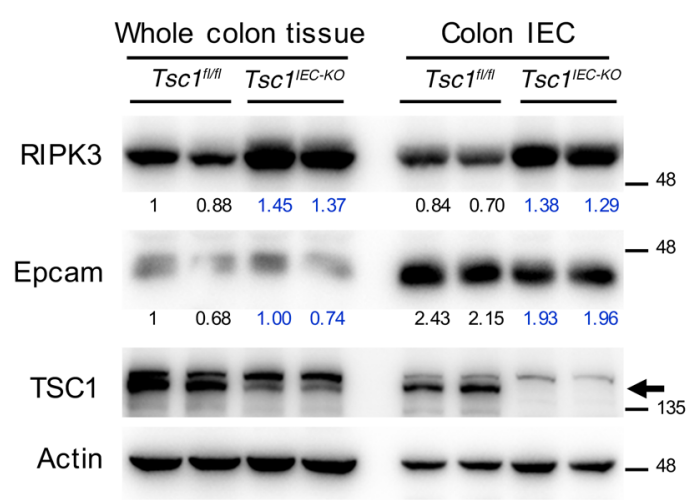

C
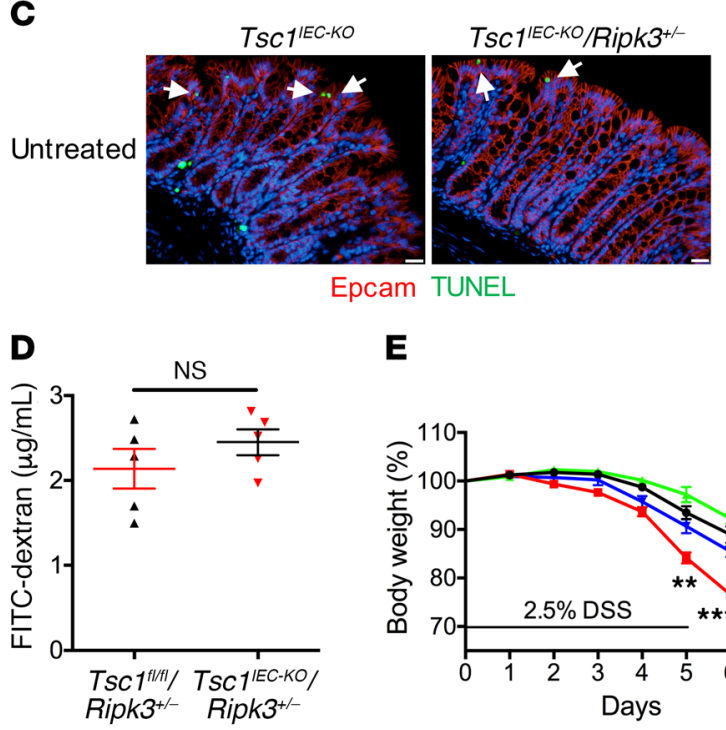

E
B
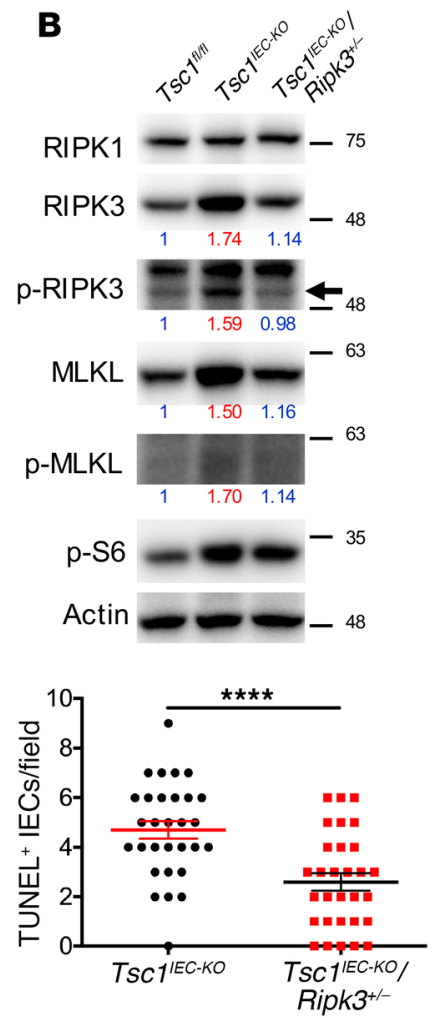

Figure 5. RIPKK accumulation correlates with severe IEC necroptosis and colitis. (A and B) Immunoblots of protein lysates from whole colon tissues or purified IECs (p-RIPK3 [S232], p-MLKL [S345], and p-S6 [S235/236]). Numbers under Western blot bands represent relative quantifications over actin. (C) IF staining and quantification of double-positive cells in colon sections $(n=6)$. Scale bars: $20 \mu \mathrm{m}$. (D) Intestinal permeability assay on $T s c f^{f / f f} / R_{i p k 3^{+/-}}$and $\operatorname{Tsc}^{1 E C-K O} / R^{2} i p k 3^{+/-}$mice $(n=5)$. (E) Mice were treated with $2.5 \%$ DSS for 5 days, and body weights were recorded daily $(n=9-12)$. Data were pooled from 3 independent experiments (E) or are representative of 3 independent experiments (A-D) and are shown as mean \pm SEM. ${ }^{* *} P<0.01,{ }^{* * * *} P<0.0001$, by unpaired Student's $t$ test.

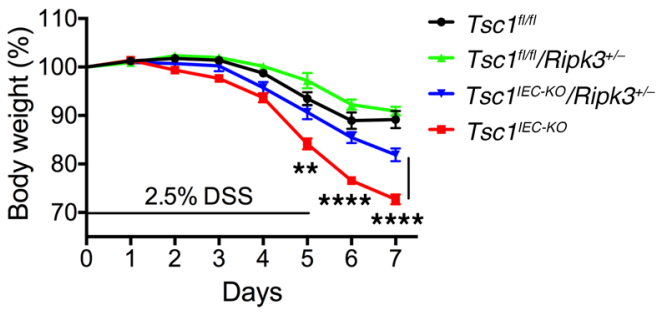

Fadd ${ }^{I E C-K O}$ IECs $(19,28)$, unlike Casp $8^{I E C-K O}$ and Fadd ${ }^{I E C-K O}$ IECs, Tsc1 ${ }^{I E C-K O}$ IECs did not upregulate the expression of Ripk3 mRNAs (Supplemental Figure 6A). Interestingly, RIPK3 accumulated in the WT organoid cells upon blockade of autophagy flux with chloroquine (CQ) or bafilomycin A1 (BFA) (Figure 6A), suggesting the involvement of autophagy in RIPK3 degradation. In contrast, with the pronounced accumulation of autophagy adaptor p62 in the steady state, the $T s c 1^{I E C-K O}$ organoid cells seemed to have only minimal autophagy flux, which was refractory to further inhibition by CQ (Figure 6A). Similarly to p62, RIPK3 proteins were highly enriched in the steady-state $T s c 1^{I E C-K O}$ organoid cells, and unchanged with CQ or BFA treatment (Figure 6A). Notably, ULK1 phosphorylation on S757, an event responsible for autophagy suppression by mTOR (40), was highly induced in Tsc $1^{I E C-K O}$ IECs, in accordance with a lower level of conversion of LC3-I to LC3-II in these cells (Figure 6B). These data further indicated a marked inhibition of autophagy flux by mTOR in Tsc1 $1^{I E C-K O}$ IECs. To extend these observations, we applied Earle's balanced salt solution (EBSS) medium, which does not contain amino acids, to induce autophagy through amino acid starvation. Remarkably, amino acid starvation induced robust autophagy as demonstrated by rapid p62 degradation in the WT cells (Figure 6C). Correspondingly, RIPK3 and MLKL, but not RIPK1, declined in WT cells following amino acid starvation (Figure 6C). Conversely, neither RIPK3 nor MLKL degraded in Tsc1-deficient cells, which were refractory to autophagy induction by amino acid starvation (Figure 6C). Furthermore, we observed prominent RIPK3 polyubiquitination, including both K48- and K63-type polyubiquitination on RIPK3, in WT cells (Figure 6D). However, RIPK3 ubiquitination, especially the K48-type polyubiquitination, was considerably decreased in Tsc1-deficient cells (Figure 6D). Consistent with the role of p62 in recognition of ubiquitinated cargo proteins for autophagy degradation, immunoprecipitation assay demonstrated that p62 was able to form complexes with RIPK3 and MLKL, but not RIPK1 (Supplemental Figure 6B). These results collectively suggest a crucial role for autophagy in the regulation of RIPK3 degradation.

Considering that Tsc1-deficient cells had less ubiquitinated RIPK3 than WT cells (Figure 6D), we reasoned that mTOR might act through an E3 ubiquitin ligase to regulate this process. To this end, we focused on TRIM family members, as many of them have been implicated in autophagy and innate immunity (58). By screening the family of TRIM proteins for their ability to mediate RIPK3 degrada- 
A

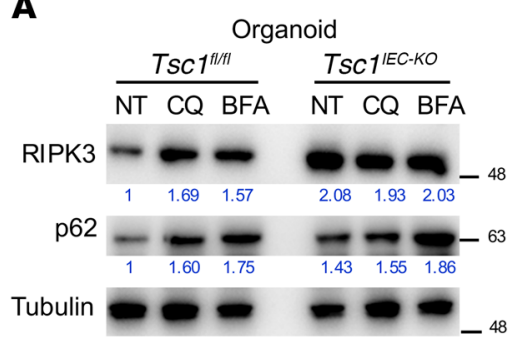

D

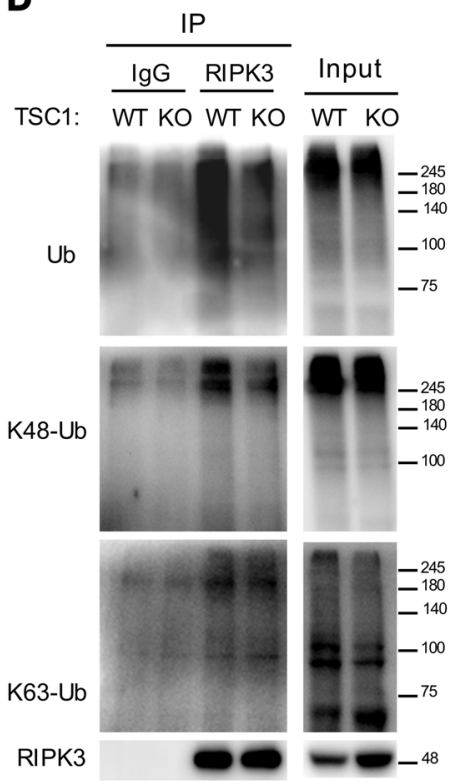

B

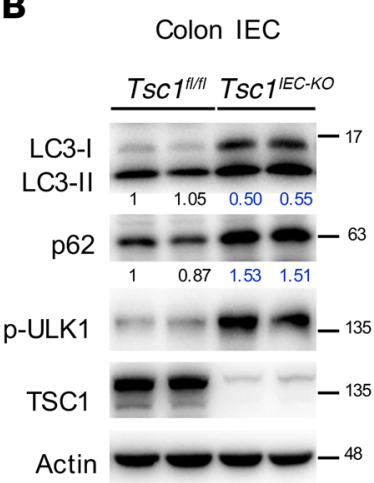

E

HA-TRIM11: Flag-RIPK3

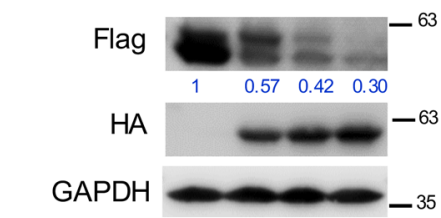

$\mathbf{F}$

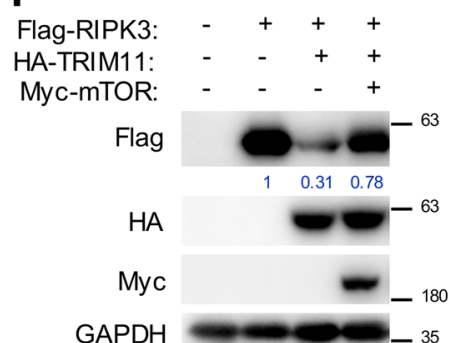

C

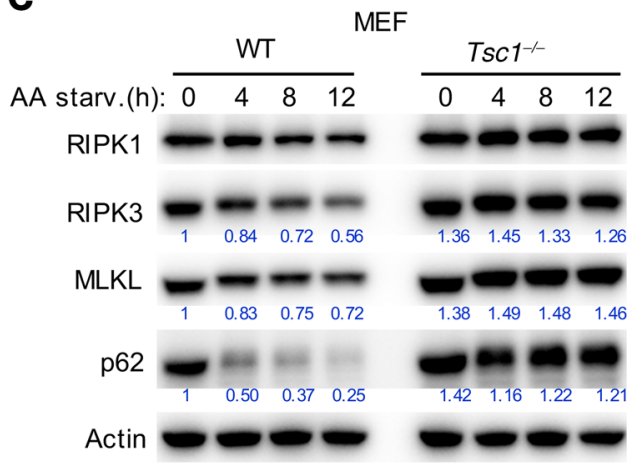

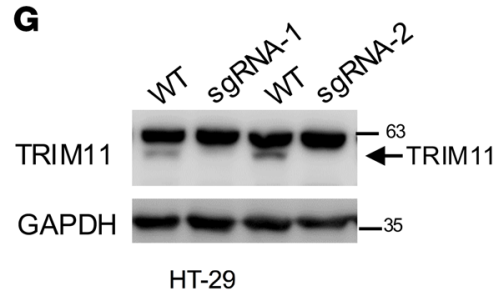

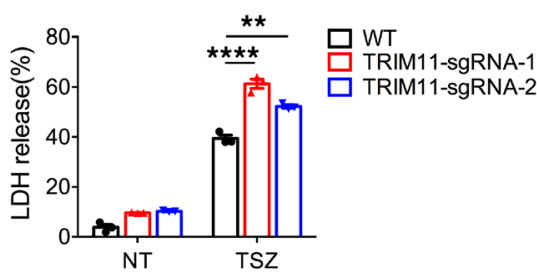

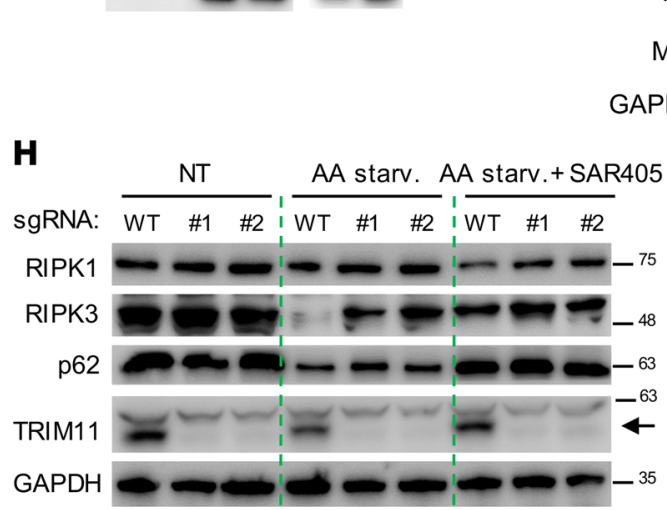
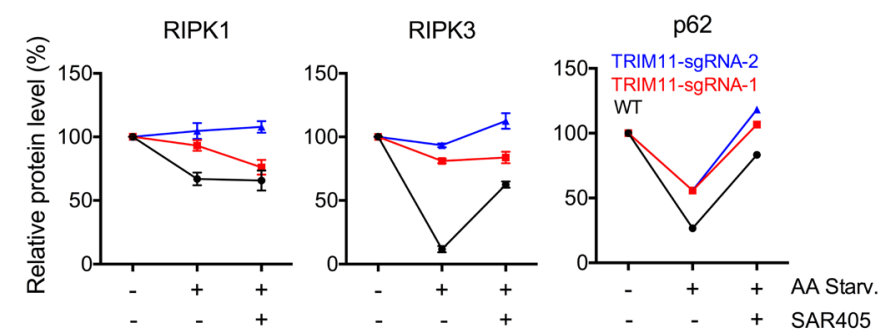

Figure 6. Autophagy and TRIM11 collaborate in RIPK3 degradation. (A) Immunoblots of protein lysates from ex vivo organoids treated with $20 \mu \mathrm{M}$ chloroquine (CQ) or $0.2 \mu \mathrm{M}$ bafilomycin A1 (BFA) for 18 hours. (B and C) Immunoblots of cell lysates from WT and Tsc 1EC-Ko IECs (B) or WT and Tsc1/- MEFs treated with amino acid-free EBSS medium for various times as indicated (p-ULK1 S757) (C). (D) Cell lysates from MEFs were immunoprecipitated with control or anti-RIPK3 antibodies, then immunoblotted with respective antibodies. (E and F) HEK293T cells were transfected with various combinations of plasmids for 36 hours. (C) WT and TRIM11-KO HT-29 cells confirmed by immunoblotting were treated with $10 \mathrm{ng} / \mathrm{mL}$ hTNF (T), $1 \mu \mathrm{M} \mathrm{BV-6}$ (S), and $20 \mu \mathrm{M}$ z-VAD (Z) for 24 hours, and cell cytotoxicity was measured by lactate dehydrogenase release ( $n=3$ biological replicates). (H) WT and TRIM11-KO HT-29 cells were treated with EBSS medium with or without $10 \mu$ M SAR405 for 24 hours. Cell lysates were immunoblotted and quantified by Imagej. Numbers to the right of Western blot bands represent relative quantifications over actin (B and $\mathbf{C})$, tubulin (A), or GAPDH (E, F, and $\mathbf{H})$. Data are representative of 3 independent experiments and are shown as mean \pm SEM. ${ }^{* *} P<0.01,{ }^{* * *} P<0.0001$, by unpaired Student's $t$ test.

tion (data not shown), we identified TRIM11 as a candidate E3 ligase for RIPK3 degradation (Figure 6E). Overexpression of WT but not the E3 ligase-inactive mutant (C56A) (59) of TRIM11 diminished RIPK3 in HT-29 cells (Supplemental Figure 6C). Moreover, overexpression of mTOR or blockade of the autophagosome with either CQ or BFA abolished TRIM11-mediated RIPK3 degradation (Figure 6F and Sup- plemental Figure 6D). Further, overexpression of mTOR decreased TRIM11-promoted auto-polyubiquitination (Supplemental Figure 6E). Collectively, these data strongly implicate the mTOR/TRIM11 axis in the regulation of the autophagic degradation of RIPK3. Next, we conducted an in vivo ubiquitination assay to test whether TRIM11 functions as an E3 ligase for RIPK3. Indeed, TRIM11 formed com- 
plexes with RIPK3 (Supplemental Figure 6F) and catalyzed notable ubiquitination on RIPK3 proteins (Supplemental Figure 6G). Hence, TRIM11 may act as an E3 ligase to regulate RIPK3 ubiquitination and degradation. To test this hypothesis, we generated Trim11 ${ }^{--}$HT-29 cells by CRISPR/Cas9 technology (Figure 6G). Remarkably, Trim11 ${ }^{--}$cells generated by 2 independent sgRNAs demonstrated increased susceptibility to TNF-induced necroptosis in the presence of $\mathrm{z}$-VAD and Smac mimetic (TSZ), hence revealing a negative role for TRIM11 in necroptosis (Figure 6G). Consistently, Trim11 ${ }^{--}$cells demonstrated elevated phosphorylation and expression of RIPK3, which led to the hyperactivation of MLKL following the stimulation with TSZ (Supplemental Figure 6H). Next, we cultured WT and Trim11 ${ }^{-/-}$cells with EBSS in combination with the Vps34 inhibitor SAR405 (60), thereby assessing the involvement of autophagy in RIPK3 degradation in these cells. Although amino acid starvation elicited similar levels of autophagy and p62 degradation in WT and Trim11 ${ }^{-/}$cells, amino acid starvation-induced RIPK3 degradation was considerably lower in the $\operatorname{Trim}_{11^{-/}}$cells (Figure $6 \mathrm{H})$. Since the Vps34 inhibitor SAR405 was able to block both p62 and RIPK3 degradation (Figure 6H), these results support the notion that TRIM11 may specifically regulate RIPK3 degradation through autophagy. Consistently, knockdown of Trim11 also led to elevated expression and phosphorylation of RIPK3 in WT mouse embryonic fibroblasts (MEFs) after stimulation with TSZ (Supplemental Figure 6I). Conversely, knockdown of Trim11 expression did not have any detectable effect on RIPK3 expression or phosphorylation in the

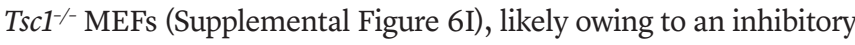
role of mTOR in Trim11 function as shown above (Figure 6F). Collectively, these results suggest that TSC1/mTOR may impinge on both selective autophagy and Trim11 in controlling RIPK3 expression and activation in the IECs.

MTOR regulates microbial PAMP- and TNF-induced necroptosis. The above observations suggest that autophagy blockade by mTOR can lead to RIPK3 accumulation and susceptibility to necroptosis. Next, we sought to explore the cytokine/microbial signals likely responsible for eliciting gut epithelium necroptosis in vivo. Although TNF and poly(I:C) have been implicated in the induction of IEC necroptosis, whether TSC1/mTOR impinges on these signals remains unclear. To this end, we used ex vivocultured enteroid cells to comprehensively investigate what necroptotic signals are responsible for IEC necroptosis in vivo. Corroborating the role of mTOR in cell growth and proliferation, Tsc1 $1^{I E C-K O}$ enteroid cells grew faster and larger than their WT counterparts (Supplemental Figure 7A). By Western blotting, we observed marked elevation of RIPK3 and MLKL expression in $T s c 1^{I E C-K O}$ enteroids, which was abrogated by rapamycin treatment (Figure 7A). In contrast, other regulators of necroptosis, especially RIPK1, CHIP, FADD, and caspase-8, were similarly expressed in $T s c 1^{I E C-K O}$ enteroids (Figure 7A). Consistently, p-RIPK3 was notably induced in the Tsc1 ${ }^{I E C-K O}$ enteroids, contingent on mTOR activation (Figure 7A). Hence, the ex vivo enteroid culture reproduced well the corresponding phenotypes manifested by in vivo IECs as described above. In the presence of the TLR3 ligand poly(I:C) or TNF alone, WT enteroids had negligible cell death; however, $T s c 1^{I E C-K O}$ enteroids demonstrated a significant extent of cell death with poly(I:C) alone (Figure 7B), implicating endogenous PAMPs as the possible trigger for necroptosis in $T s c 1^{I E C-K O}$ colon. With the presence of the broad-spectrum caspase inhibitor $\mathrm{z}-\mathrm{VAD}$, poly(I:C), TNF, and IFN- $\beta$ all triggered robust cell death in both types of enteroids; nevertheless, Ts $C 1^{I E C-K O}$ enteroids showed more pronounced cell death, which was blunted by Ripk3 heterozygosity (Figure 7C). In addition to poly(I:C), the TLR ligands LPS, flagellin, and MDP also triggered much severe cell death in Tsc1 ${ }^{I E C-K O}$ enteroid cells, as assessed by propidium iodide staining (Supplemental Figure 7B). Hence, Tsc1 ${ }^{I E C-K O}$ enteroids are highly susceptible to a variety of necroptosis-inducing signals, particularly poly(I:C) and TNF.

Next, we extended our study to MEF and HT-29, 2 cell lines widely used for necroptosis study. As previously reported $(17,18)$, TNF was able to induce necroptosis in both MEF and HT-29 cells in the presence of z-VAD and Smac mimetic. Notably, knockout or knockdown of Tsc1 considerably increased TNF-induced necroptosis in these cells (Figure 7D and Supplemental Figure 7C). Similarly, poly(I:C) stimulation also triggered robust necroptosis in Tsc1-knockout MEFs (Figure 7D). It is noteworthy that RIPK3 but not RIPK1 expression was also elevated in Tsc1-knockout cells, revealing a general role for TSC1/mTOR in the regulation of RIPK3 expression (Figure 7E). Consistently, Tsc1 deficiency also led to a substantial elevation in RIPK3 and MLKL phosphorylation, without significant influence on RIPK1 upon poly(I:C) or TNF stimulation (Figure 7, E and F). Surprisingly, Tsc1 deficiency did not have a significant impact on TNF-induced activation of NF- $\kappa \mathrm{B}$ and MAPKs, except that p38 activation was somewhat impaired (Figure $7 G$ ). Together, these results demonstrate that the TSC1/mTOR axis participates in the regulation of RIPK3 expression and activation in a variety of necroptosis-inducing signals, especially poly(I:C) and TNF.

The $m T O R / R I P K 3 /$ necroptosis axis promotes chronic inflammation and cancer. Chronic inflammation, a hallmark of IBD and a driving force for human cancers, especially colon cancers, has been intrinsically associated with necroptosis. In this regard, we went on to interrogate how epithelium mTOR-regulated necroptosis influences chronic inflammation and colon cancer. Repeated DSS challenge induces chronic inflammation in the colon, which can drastically promote tumor development in conjunction with the carcinogen azoxymethane (AOM). Although a standard regimen was previously used by us (61) to induce colitis-associated colon cancer, here we modified the DSS treatment strategy to accommodate the hypersusceptibility of Ts $C 1^{I E C-K O}$ mice (Supplemental Figure 8A). Despite being challenged with a less rigorous regimen (1.5\%, $1.5 \%$, and $1 \%$ of DSS, respectively), Tsc1 ${ }^{1 E C-K O}$ mice still developed robust inflammation and suffered extended morbidity and mortality (Supplemental Figure 8B). Although all the WT mice survived to the end of the regimen, approximately $60 \%$ of $T s c 1^{I E C-K O}$ mice died of overt inflammation during treatment (Supplemental Figure 8B).

Analyzing the surviving mice, we noticed prominent colonic tumorigenesis in Tsc1 ${ }^{I E C-K O}$ mice, which had nearly 2-fold more tumors per colon (averaging 14 vs. 5 tumors per colon) than WT mice (Figure 8A and Supplemental Figure 8C). Moreover, Tsc ${ }^{I E C-K O}$ mice also had 5 times more large tumors (15\% vs. $3 \%$ ) than WT mice (Figure $8 \mathrm{~A}$ ). Besides, histological analysis revealed a high degree of dysplasia in the $T s c 1^{I E C-K O}$ tumors (Figure $8 \mathrm{~B}$ ), indicating accelerated tumor progression. Compared with the WT tumors, 
A

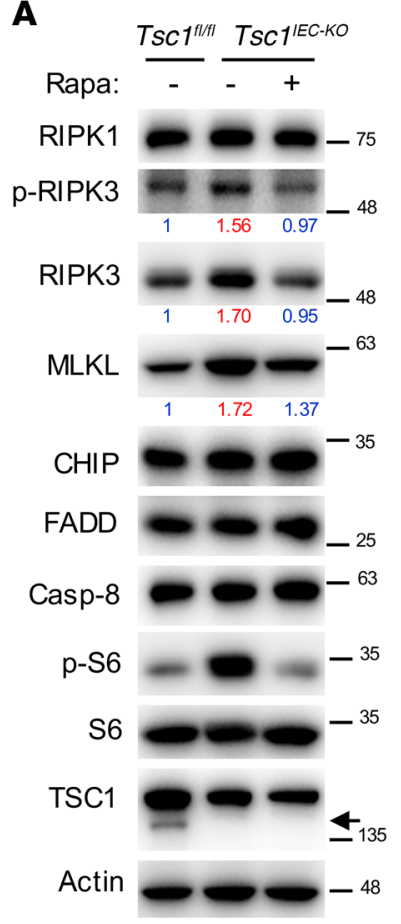

B

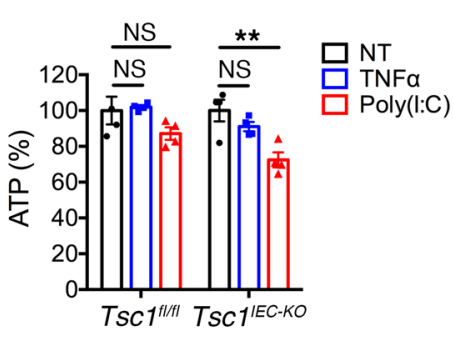

C

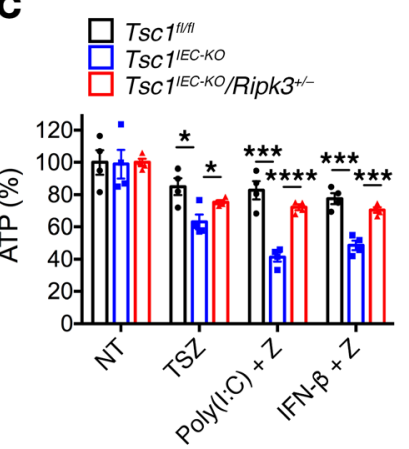

D

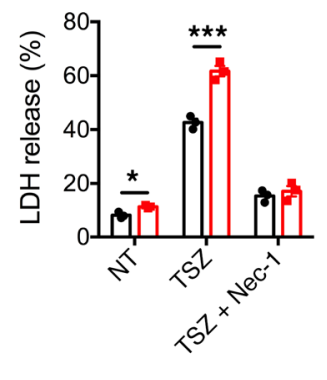

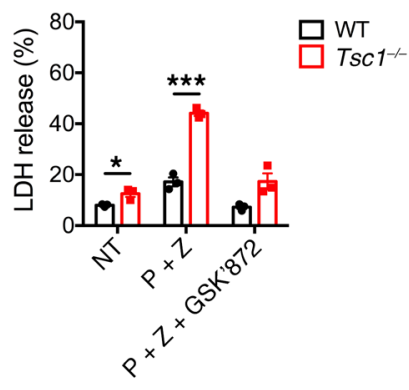

E

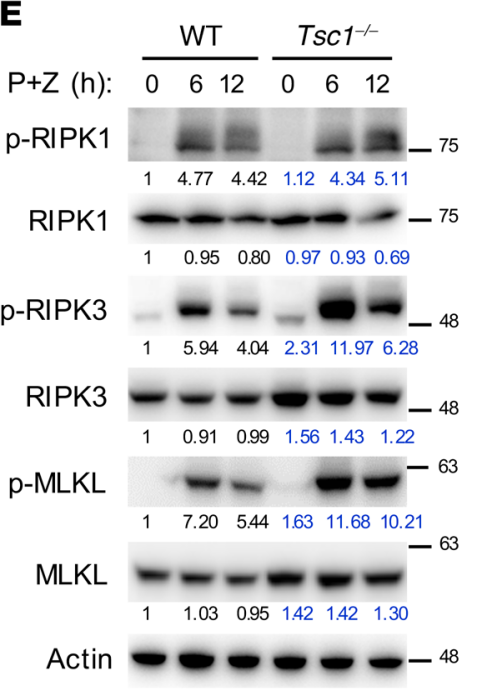

$\mathbf{F}$

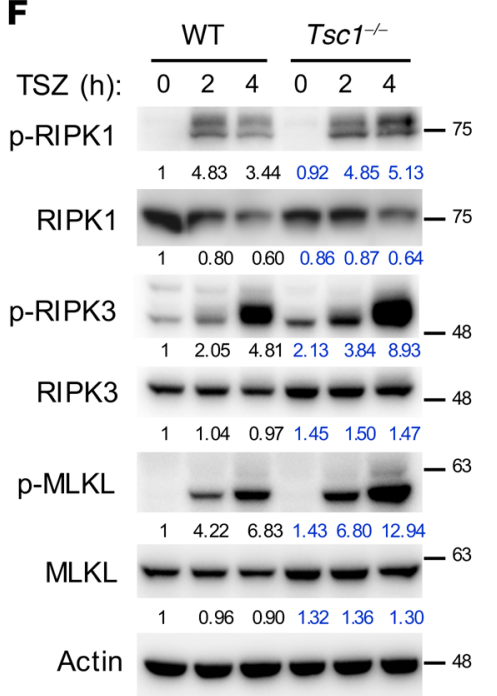

G

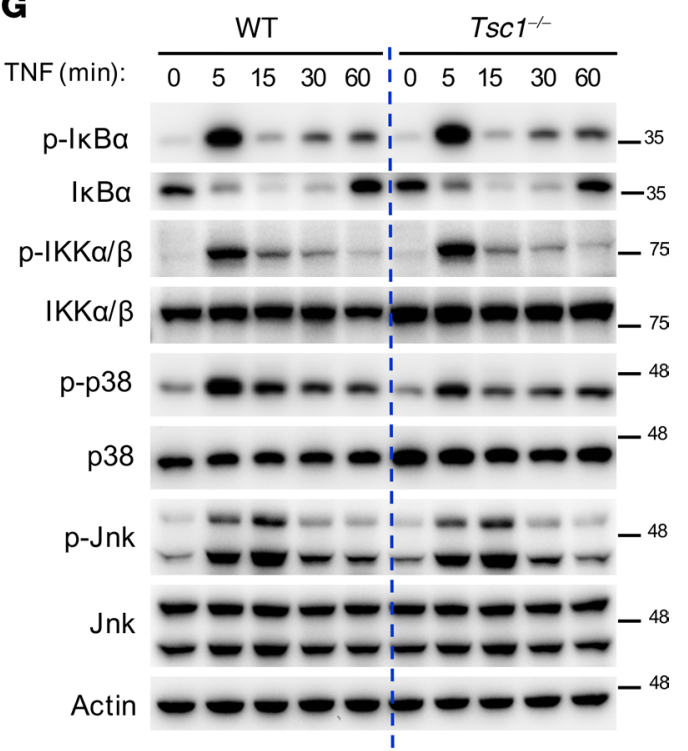

Figure 7. mTOR regulates microbial PAMP- and TNF-induced necroptosis. (A) Immunoblots of protein lysates of ex vivo organoids treated with $10 \mathrm{ng} / \mathrm{mL}$ rapamycin for 48 hours. (B and C) Organoid viability was quantified by cellular ATP levels after treatment with $10 \mathrm{ng} / \mathrm{mL} \mathrm{mTNF}$ or $10 \mu \mathrm{g} / \mathrm{mL}$ poly $(\mathrm{I}: \mathrm{C})$ alone

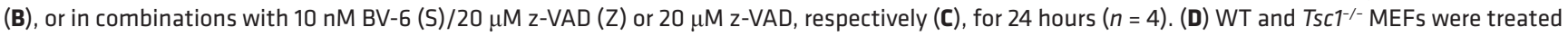

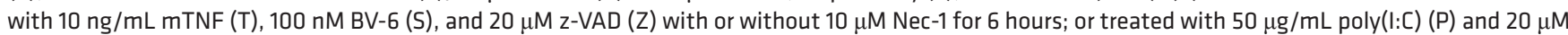
z-VAD (Z) with or without $5 \mu$ M CSK' 872 for $16-20$ hours. Cell cytotoxicity was measured by LDH release $(n=3)$. (E-C) MEFs were treated with $50 \mu g / m L$ poly(I:C) (P) and $20 \mu \mathrm{M} \mathrm{z-VAD} \mathrm{(Z)} \mathrm{(E)} \mathrm{or} 10 \mathrm{ng} / \mathrm{mL}$ mTNF (T), $100 \mathrm{nM} \mathrm{BV-6}$ (S), and $20 \mu \mathrm{M} \mathrm{z-VAD} \mathrm{(Z)} \mathrm{(F)} \mathrm{or} 10 \mathrm{ng} / \mathrm{mL}$ mTNF alone (C) for various times as

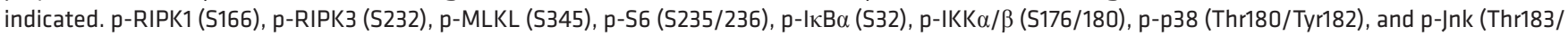
Tyr185) were used. Numbers under Western blot bands represent relative quantifications over actin. Data are representative of 3 independent experiments and are shown as mean $\pm \mathrm{SEM}$. ${ }^{*} P<0.05,{ }^{* *} P<0.01,{ }^{* *} P<0.001$, ${ }^{* * *} P<0.0001$, by unpaired Student's $t$ test.

the $T s c 1^{I E C-K O}$ tumors showed much stronger p-S6 staining (Figure 8C), as well as abundant accumulation of RIPK3 proteins (Figure 8D). Regardless of genotypes, all the tumors showed much higher RIPK3 expression than the respective normal colon tissues (Figure 8, D and E). Consistent with elevated p-RIPK3 and p-MLKL in $T s c 1^{I E C-K O}$ tumors (Figure 8E), more necrotic cells were presented in $T s c 1^{I E C-K O}$ tumors than in WT tumors (Figure $8 \mathrm{~F}$ ). Moreover, $T s c 1^{I E C-K O}$ tumors showed a drastic increase in the induction of the proinflammatory mediators Cox2, Mmp1O, and Ereg, as well as the cytokines $I l 1 b$, Il6, and Il22 (Supplemental Figure 8D). Together, these results revealed that colon cancer is associated with hyperactive mTOR and profound necroptosis. 
Considering that both tumor-suppressive and tumor-promoting roles of RIPK3 and necroptosis have been reported in various cancers $(34,62,63)$, we used $T s c 1^{I E C-K O} / R_{i p k 3^{+/-}}$mice to further assess the role of RIPK 3 and necroptosis in colitis-associated colon cancer. As compared with Ts $I^{I E C-K O}$ tumors, we observed a profound reduction in inflammatory cytokines/mediators in $T s c 1^{I E C-K O}$ / Ripk3 $^{+/}$tumors (Figure 9A), suggesting a critical role for RIPK3 in driving the overt inflammation in $T s c 1^{I E C-K O}$ tumors. Correspondingly, Tsc1 ${ }^{I E C-K O} /$ Ripk3 $^{+/-}$mice exhibited decreased tumorigenesis, with fewer tumors per mouse than $T s c 1^{I E C-K O}$ mice (Figure 9B). In contrast, Ripk3 $^{+/}$mice exhibited a slight increase in tumor number compared with WT mice (Figure 9B), corroborating increased tumorigenesis in Ripk $^{-1-}$ mice in previous reports $(34,63)$. It is important to note that while Tsc1 IEC deficiency led to a substantial increase in colon cancer development in WT mice $(\sim 2.5$-fold), its tumor-promoting effect was considerably dampened in Ripk3+/mice ( 1.7-fold) (Figure 9B). Besides, Tsc1 $1^{\mathrm{IEC}-\mathrm{KO}} / \mathrm{Ripk}^{+/-}$tumors exhibited a lower level of epithelial necroptosis than Tsc1 ${ }^{I E C-K O}$ tumors (Figure 9C), revealing a positive correlation between necroptosis and colonic tumorigenesis. Collectively, these data support a tumor-promoting role for epithelial RIPK3 and necroptosis in colitis-associated colon cancer.

\section{Discussion}

Here we propose that epithelial mTOR functions as a metabolic checkpoint for intestinal dysfunction and inflammation through the integration of dietary and microbial cues in the gut. Compelling evidence from this study firstly demonstrates that epithelial mTOR hyperactivation, an event frequently associated with the Western diet, dysbiosis, and genetic alterations, is intimately linked to aberrant RIPK3-MLKL activation, overt IEC necroptosis, and barrier dysfunction. Further, our results also implicate the epithelial mTOR/RIPK3/necroptosis axis as a vicious circuit in the pathogenesis of IBD and cancer. Therefore, we believe that through a constant survey of environmental signals, epithelium mTOR might operate as a rheostat balancing autophagy and necroptosis, 2 processes critically involved in intestinal homeostasis and inflammation (Supplemental Figure 9).

Epithelium homeostasis rests on the dynamic equilibrium of cell proliferation and death, and aberrant cell death can lead to intestinal barrier disruption and inflammation. Along with apoptosis, necroptosis has emerged as a critical player in the regulation of intestinal homeostasis (13). Although mTOR has been previously linked to intestinal epithelium growth and proliferation $(42-44,64)$, here we discovered an unexpected role for mTOR in epithelium necroptosis. Overall, studies from others $(41,44)$ and us strongly suggest that precision control of mTOR activity in the gut epithelium is crucial for intestinal homeostasis. In support of this notion, we found that mTOR hyperactivation triggered by the Western diet or genetic ablation of Tsc1 was able to tilt the balance from pro-cell growth toward pro-cell death, thereby shifting intestinal homeostasis to barrier disruption and inflammation. Moreover, in human IBDs, aberrant mTOR activation and prominent necroptosis were intertwined and were intimately associated with overt inflammation. Hence, our results identify mTOR as a metabolic hub linking environmental risk factors to epithelium disequilibrium, thereby provid- ing a molecular explanation for the epidemiological association of the Western diet with IBD.

Although RIPK3 expression can be regulated via both transcriptional and post-transcriptional mechanisms, our results indicate that mTOR primarily regulates RIPK3 at the post-transcriptional level. It has been reported that a fraction of polyubiquitinated RIPK3 protein undergoes rapid degradation by the proteasome (65). Moreover, RIPK3 protein ubiquitinated by the E3 ligase CHIP can also be degraded by lysosome (27). Here, our results suggest selective autophagy as a novel mechanism mediating RIPK3 degradation, thus adding another layer of regulation on RIPK3. Under amino acid-restricted conditions, autophagy can be induced to promote rapid RIPK3 degradation. Conversely, amino acid surplus or Tsc1 deficiency led to mTOR hyperactivation and ULK1 phosphorylation, which resulted in autophagy shutdown and ensuing RIPK3 accumulation. Additionally, we identified TRIM11 as an E3 ubiquitin ligase responsible for RIPK3 polyubiquitination and autophagy degradation. Although TRIM11 has been previously linked to the degradation of misfolded polyQ and AIM2 $(59,66)$, its role in necroptosis has not been implicated. Our data suggest that TRIM11 likely primarily assembles K48-type polyubiquitin chains on RIPK3, a marker that could be recognized by autophagy adaptor p62 for autophagosome degradation. Interestingly, mTOR appears to be able to counteract TRIM11-mediated ubiquitination and degradation of RIPK3, although the precise mechanism remains to be elucidated. Hence, our study suggests that mTOR may prevent RIPK3 degradation through 2 interrelated mechanisms that converge on autophagy degradation of RIPK3 proteins.

In addition to rapamycin, we found that microbiota depletion considerably ameliorated RIPK3 accumulation and activation in Tsc1-deficient IECs. These findings collectively suggest that epithelium TSC1/mTOR has a crucial role in responding to microbiota, by maintaining a delicate balance between autophagy and necroptosis in the gut. It is important to note that while MLKL expression is mostly regulated independently of RIPK3 $(18,52)$, we observed concomitant elevation of MLKL and RIPK3 in Tsc1-deficient as well as Western diet-treated IECs. Given that RIPK3 and MLKL can also execute necroptosisindependent functions $(13,34,52)$, the coregulation of both together reinforces the notion that the TSC1/mTOR pathway has a designated role in necroptosis. Nevertheless, whether autophagy has a similar role in MLKL regulation remains unknown at present, but certainly warrants further investigation. Interestingly, a recent study reports that Atg16L1-deficient Paneth cells are hypersusceptible to TNF-triggered necroptosis (67), thus providing another paradigm for the intricate interplay of autophagy and necroptosis. Hence, we propose that autophagy may serve as a checkpoint for necroptosis in controlling intestinal homeostasis and inflammation.

Despite the intrinsic link of necroptosis to barrier disruption and inflammation, 2 hallmarks of IBD, the role of necroptosis in colitis has yet to be unequivocally established. Although blockade of RIPK1 by Nec-1 led to attenuated DSS-induced colitis (68), thus suggesting a proinflammatory role for necroptosis, studies on Ripk3-knockout mice failed to support this conclusion $(30,33)$. Instead, a protective role executed by the CD11 $\mathrm{c}^{\mathrm{hi}}$ mononuclear 
A
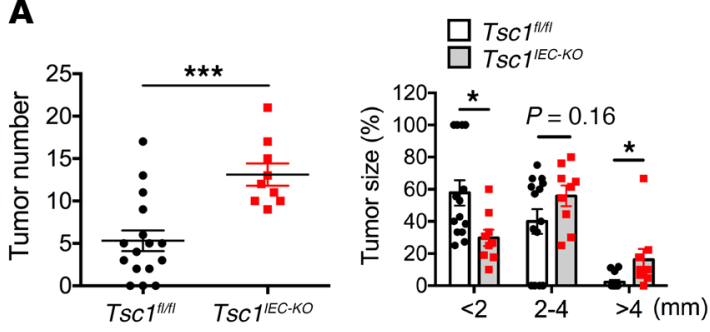

C

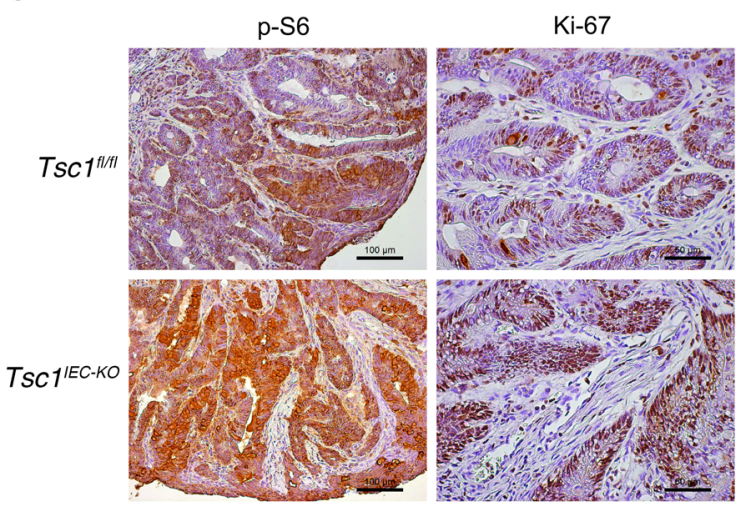

E

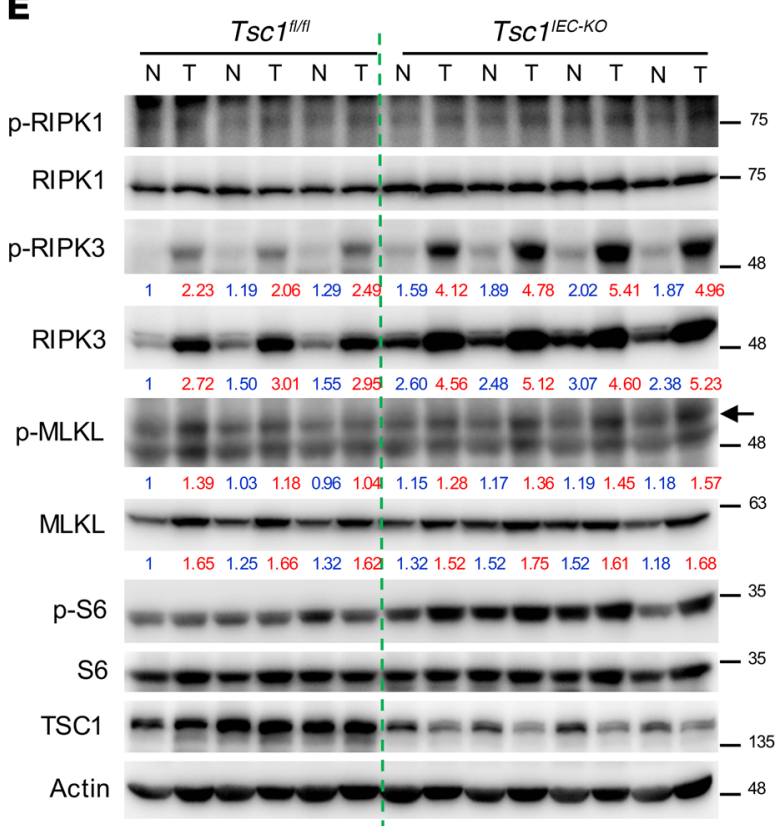

B

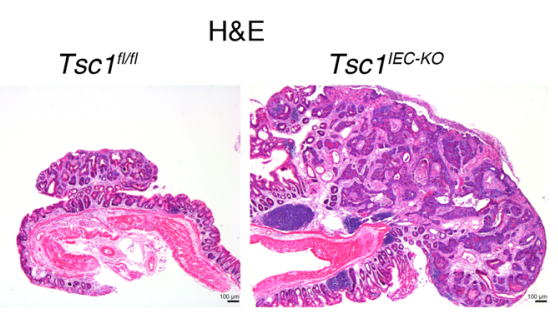

D

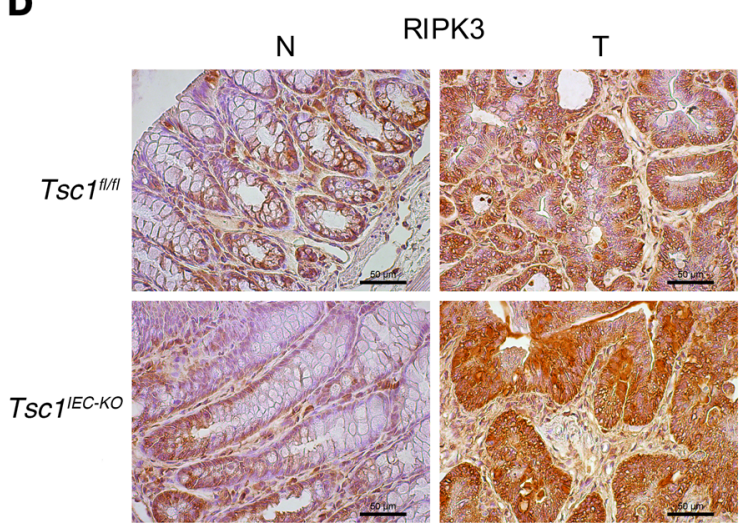

$\mathbf{F}$
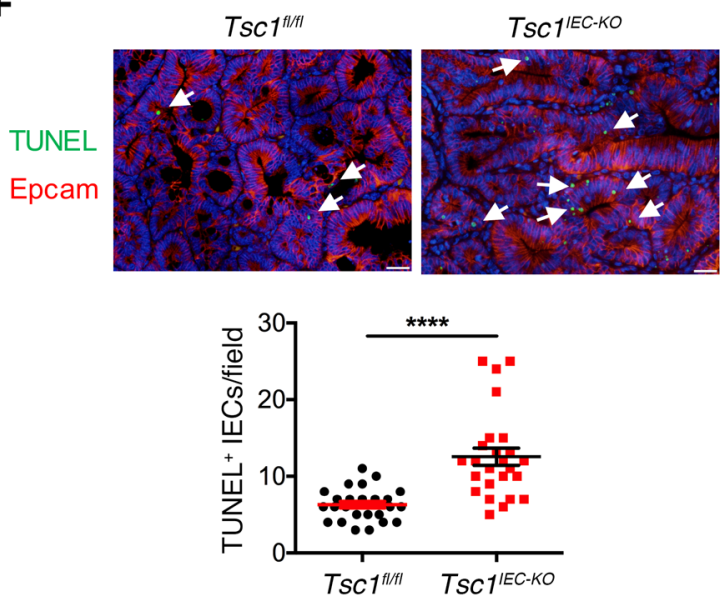

Figure 8. mTOR regulates RIPK3 overexpression and necroptosis in colitis-associated cancers. (A) Tumor number and volume were assessed in Tsc $7^{f l / f l}$ and $T S{ }^{1 E C-K O}$ mice challenged with the colitis-associated colon cancer (CAC) regimen for 80 days $(n=9-16)$. (B-D) H\&E and IHC staining of colon tumors from CAC-challenged mice. Scale bars: $100 \mu \mathrm{m}$ (H\&E and p-S6), $50 \mu \mathrm{m}$ (Ki-67 and RIPK3). (E) Immunoblots of protein lysates from colon tumors (T) or adjacent normal colon tissues (N) of CAC-challenged mice $(n=3-4)$. p-RIPK1 (S166), p-RIPK3 (S232), p-MLKL (S345), and p-S6 (S235/236) were used. Numbers under Western blot bands represent relative quantifications over actin. (F) IF staining and quantification of Epcam/TUNEL-double positive cells in colon tumor sections of CAC-challenged mice $(n=5)$. Scale bars: $20 \mu \mathrm{m}$. Data were pooled from 2 independent experiments $(\mathbf{A})$ or are representative of 2 independent experiments (E and $\mathbf{F}$ ) and are shown as mean $\pm \mathrm{SEM} .{ }^{*} P<0.05$, ${ }^{* *} P<0.001$, ${ }^{* * *} P<0.0001$, by unpaired Student's $t$ test.

phagocytes has been suggested for RIPK3 (30, 31). Although RIPK3 tends to execute necroptosis-independent roles in the immune cell compartment, studies from others $(19,28)$ and us strongly suggest that RIPK3 is reserved predominantly for necroptosis in the IECs. Genetic ablation of either Tsc1, Casp8, or Fadd in IECs led to RIPK3 accumulation, led to aberrant epithelium necrosis, and exacerbated experimental or spontaneous colitis. Although others $(19,28,51,69)$ have shown that pharmacological inhibition or genetic ablation of Ripk3 in Casp $8^{I E C-K O}$ and Fadd ${ }^{I E C-K O}$ mice alleviated epithelium death and colitis, our data demonstrate that genetic depletion of Ripk3 or $M l k l$ was able to ameliorate epithelium necrosis, restoring barrier function and repressing colitis 
A
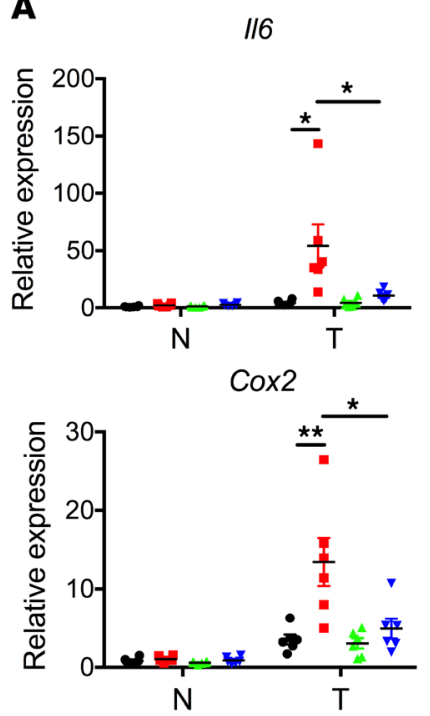

B

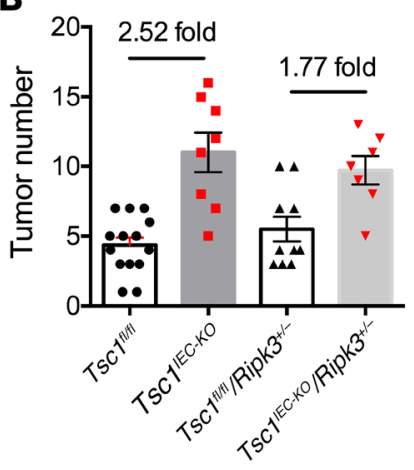

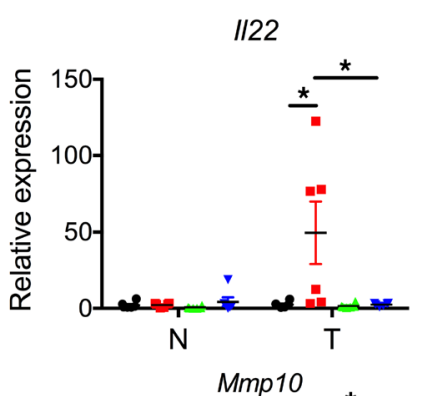

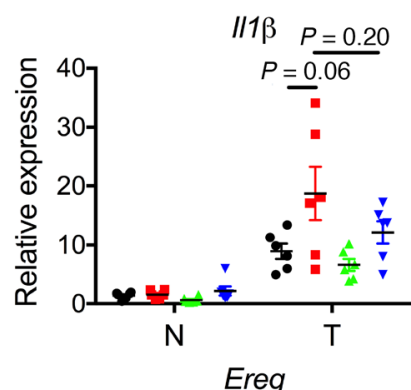

- $T s c 1^{\mathrm{fi} / \mathrm{f}}$

- TsC1'EC-KO

A Tsc1 1/r//Ripk3+1-

- TsC1 1EC-KO/Ripk $\widehat{T}^{+1}$
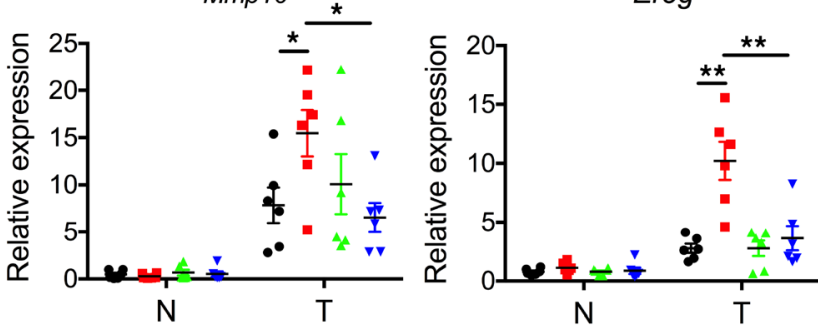

C

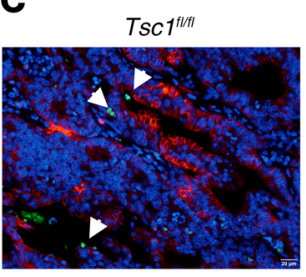

Tsc1 filfl/Ripk3 $^{+1}$

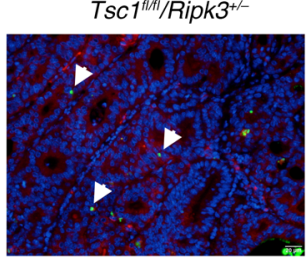

Epcam TUNEL

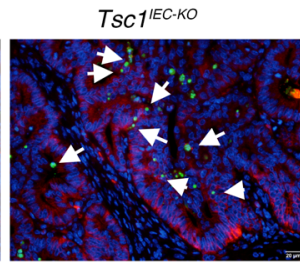

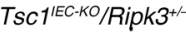

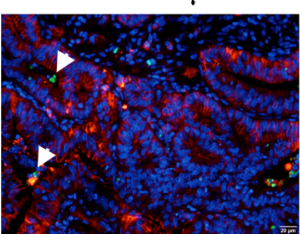

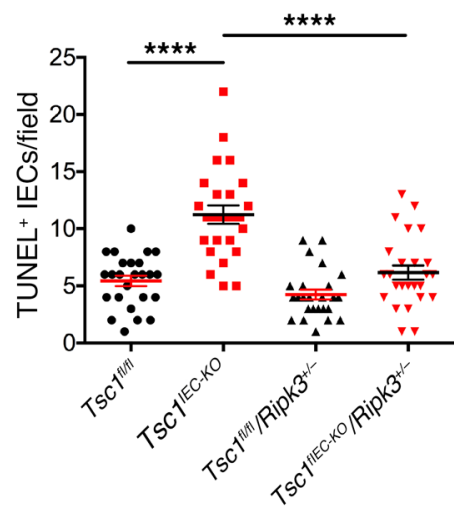

Figure 9. RIPK3 promotes inflammation and necroptosis in Tsc1 ${ }^{1 E-K o}$ colon cancers. (A) qPCR analysis of proinflammatory gene expression (normalized to $\beta$-actin) in colon tumors $(T)$ and normal colon tissues $(N)$ following the CAC regimen $(n=6)$. (B) Tumor numbers formed in various genotypes of mice upon CAC challenge $(n=7-13)$. (C) IF staining and quantification of double-positive cells in colon tumor sections $(n=25)$. Scale bars: $20 \mu \mathrm{m}$. Data were pooled from 2 independent experiments (B) or are representative of 2 independent experiments $\left(\mathbf{A}\right.$ and $\mathbf{C}$ ) and are shown as mean $\pm \mathrm{SEM}$. ${ }^{*} P<0.05$, ${ }^{*} P<0.01$, ${ }^{* * * *} P<0.0001$ by 1 -way ANOVA.

in $T s c 1^{I E C-K O}$ mice. Collectively, these observations uncover a causative role for epithelium RIPK3 and MLKL in intestinal homeostasis and colitis. Additionally, we validated a strong and widespread correlation between mTOR activation and epithelium necroptosis in human IBDs, hence providing insight into the widespread association of IBD with the Western diet. With all these pieces of evidence, we propose that in the gut epithelium, RIPK3 and necroptosis may form a bad liaison driving IBD pathogenesis and thus should be considered as therapeutic targets in the future.

The role of necroptosis in cancer appears complex; while a large number of studies implicate RIPK3 as a tumor suppressor (62), a tumor-promoting role for RIPK3 has also emerged, especially in pancreatic cancer and intrahepatic cholangiocarcinoma $(70,71)$. The fact that RIPK3 is shut down in most cancer cell lines has led to the popular view that RIPK 3 and necroptosis are incompatible with cancer development $(17,62,72,73)$. Corroborating this hypothesis, Ripk3-knockout mice showed increased tumorigenesis following AOM plus DSS treatment $(34,63)$. Paradoxically, we and others (74) observed an increase rather than a decrease of RIPK3 expression in colon tumors induced by AOM plus DSS regimen or $A p c^{m i n}$ heterozygosity. Moreover, the ablation of 1 allele of Ripk3 in Tsc $1^{I E C-K O}$ mice led to decreased tumorigenesis and concomitant reduction in necroptosis and inflammation. Hence, it is plausible that RIPK3-promoted necroptosis might have contributed to the overt chronic inflammation in Ts $1^{I E C-К O ~ m i c e, ~ t h e r e b y ~ d r i v i n g ~ c o m p e n s a t o r y ~ c e l l ~ p r o l i f e r a t i o n ~}$ to accelerate tumor progression. Although more studies are certainly required to clarify the role of RIPK3 in tumorigenesis, our study nonetheless suggests that RIPK3 can be upregulated in a subset of cancers with mTOR dysregulation and thus should be considered as a therapeutic target. As a proof of principle, Smac mimetic has been shown to be effective in the induction of necroptosis and treatment of RIPK3-high colon cancers and acute myeloid leukemias $(74,75)$. Considering that mTOR dys- 
regulation is widely associated with human cancers, it will be of great interest to explore how to harness RIPK3 overexpression for cancer therapy in individuals with gain-of-function $\mathrm{mTOR}$ or loss-of-function caspase-8.

In summary, our results present the $\mathrm{TSC} 1 / \mathrm{mTOR}$ axis as a node of the complex immune/metabolic network in the gut epithelium, delicately balancing autophagy and necroptosis via integrating environmental cues. We also provide compelling evidence suggesting mTOR and necroptosis as underpinnings of Western diet/dysbiosis-triggered intestinal dysfunction, inflammation, and cancer. Hence, we propose that RIPK3 and necroptosis should be considered as a potential target for the treatment of IBD and colon cancer.

\section{Methods}

Supplemental Methods are available online with this article; https:// doi.org/10.1172/JCI133264DS1.

Mice. Tsc1-floxed (76), mTor-floxed (77), and Villin-cre transgenic mice (78) were purchased from The Jackson Laboratory. Ripk3 $3^{--}$and $\mathrm{Mlkl}^{-/-}$mice on C57BL/6 background were described previously (23, 52). Tsc1-floxed mice were bred onto Villin-cre mice to generate intestinal epithelium-specific Tsc1-knockout mice, Tsc1 $1^{f / f l}$ Villin $^{\text {cre }}\left(T s c 1^{I E C-K O}\right)$. $T s c 1^{I E C-K O}$ mice were backcrossed to WT C57BL/6 mice for 6 generations. In all experiments, littermates carrying loxP-flanked alleles but without Cre recombinase were used as controls $\left(T s c 1^{f / f f}\right) \cdot m \operatorname{Tor}^{f / f l}$, Ripk3-/, and $\mathrm{Mlkl}^{-/-}$mice were bred onto $T s c 1^{I E C-K O}$ mice at least 3 generations to generate compound mutant mice. For dietary experiments, mice were fed with a standard diet (D10012G, Research Diets) that contains 20\% protein $/ 16 \%$ fat, a high-protein diet comprising $60 \%$ protein $/ 16 \%$ fat, or a high-fat diet with $20 \%$ protein/60\% fat (D12492, Research Diets). All the mice were bred and maintained in a specific pathogen-free animal facility at Institut Pasteur of Shanghai, except for the germ-free C57BL/6 mice, which were bred and maintained in germ-free conditions in vinyl isolators of the animal facility at the Third Military Medical University.

Human samples. Paraffin-embedded specimens of colonic mucosae were obtained from control individuals $(n=14)$ or active Crohn's disease patients $(n=14)$ at the First Affiliated Hospital of Sun Yat-sen University. The diagnosis of Crohn's disease was based on a standard combination of clinical, endoscopic, and histological criteria.

Induction of colitis and clinical score. Age- and sex-matched 8- to 10-week-old littermates were challenged with DSS (MP Biomedicals; MW 36,000-50,000) for 5 days in drinking water to induce colitis. Unless mentioned otherwise, mice were treated with $2.5 \%$ DSS. All the mice were cohoused before and during colitis induction, and littermates were used for experiments whenever possible. After a 5-day
DSS treatment, mice were given regular water until the end of the experiment. For rapamycin treatment, mice were injected i.p. with $5 \mathrm{mg} / \mathrm{kg}$ rapamycin dissolved in PBS with $5 \%$ Tween- 80 for 5 days (3 days before DSS treatment and 2 days after DSS treatment). DSSinduced clinical scores were determined by average scores of weight loss, bleeding, and stool condition as described previously (79). Briefly, for weight loss: no weight loss was scored 0, $1 \%-5 \%$ weight loss scored 1, 5\%-10\% weight loss scored 2, 10\%-20\% weight loss scored 3, and more than $20 \%$ weight loss scored 4 . For bleeding, negative hemoccult was scored 0 , positive hemoccult scored 2 , and gross bleeding scored 4 . For stool, well-formed pellets were scored 0 , pasty and semiformed stools scored 2, and liquid stools scored 4 .

Statistics. Survival curve was analyzed by log-rank (Mantel-Cox) test. Unpaired 2-tailed Student's $t$ test was used to compare 2 groups; 1 -way ANOVA was used for multiple groups. $P$ less than 0.05 was considered significant.

Study approval. Written patient consent was provided, and ethical approval for the use of human samples was granted by the Ethical Committee of the First Affiliated Hospital of Sun Yat-sen University before collection of human tissue samples. All the animal studies were conducted in compliance with the protocol approved by the Institutional Animal Care and Use Committee at Institut Pasteur of Shanghai.

\section{Author contributions}

YX, HX, and HL designed the experiments. YX, YZ, WL, ML, K Chen, XC, LS, TL, and DS performed the experiments. YX, YZ, $\mathrm{K}$ Chen, LS, and HX analyzed the data. HZ, HZ, ZK, YMI, XY, K Cadwell, JL, HW, YC, XL, XZ, JC, SC, QL, and HL provided important materials and technical support. YX and HX wrote the manuscript.

\section{Acknowledgments}

This study was supported by the National Natural Science Foundation of China (81720108019, 81725004, 91542206, 31470847, and 31700784), the Key development and research project 2016YFA0502100, the Strategic Priority Research Program of the Chinese Academy of Sciences (XDB29030302), and the Shanghai Municipal Science and Technology Major Project (2019SHZDZX02).

Address correspondence to: Hui Xiao, Institut Pasteur of Shanghai, 320 Yueyang Road, Shanghai 200031, China. Phone: 86.21.5492.3127; Email: huixiao@ips.ac.cn. Or to: Huabin Li, Affiliated Eye, Ear, Nose and Throat Hospital, Fudan University, 83 Fenyang Road, Shanghai 200031, China. Phone: 86.21.6437.7134; Email: allergyli@163.com.
1. de Souza HS, Fiocchi C. Immunopathogenesis of IBD: current state of the art. Nat Rev Gastroenterol Hepatol. 2016;13(1):13-27.

2. Hall AB, Tolonen AC, Xavier RJ. Human genetic variation and the gut microbiome in disease. Nat Rev Genet. 2017;18(11):690-699.

3. Kaplan GG, Ng SC. Understanding and preventing the global increase of inflammatory bowel disease. Gastroenterology. 2017;152(2):313-321.e2.

4. Lee D, et al. Diet in the pathogenesis and treatment of inflammatory bowel diseases. Gastroenterology. 2015;148(6):1087-1106.
5. Thorburn AN, Macia L, Mackay CR. Diet, metabolites, and "western-lifestyle" inflammatory diseases. Immunity. 2014;40(6):833-842.

6. Uniken Venema WT, Voskuil MD, Dijkstra G, Weersma RK, Festen EA. The genetic background of inflammatory bowel disease: from correlation to causality. J Pathol. 2017;241(2):146-158.

7. Adolph TE, et al. Paneth cells as a site of origin for intestinal inflammation. Nature. 2013;503(7475):272-276.

8. Baxt LA, Xavier RJ. Role of autophagy in the maintenance of intestinal homeostasis. Gastroen- terology. 2015;149(3):553-562.

9. Cadwell K. Crosstalk between autophagy and inflammatory signalling pathways: balancing defence and homeostasis. Nat Rev Immunol. 2016;16(11):661-675.

10. Lewis JD, Abreu MT. Diet as a trigger or therapy for inflammatory bowel diseases. Gastroenterology. 2017;152(2):398-414.e6.

11. Dannappel M, et al. RIPK1 maintains epithelial homeostasis by inhibiting apoptosis and necroptosis. Nature. 2014;513(7516):90-94.

12. Takahashi N, et al. RIPK1 ensures intestinal 
homeostasis by protecting the epithelium against apoptosis. Nature. 2014;513(7516):95-99.

13. Pasparakis M, Vandenabeele P. Necroptosis and its role in inflammation. Nature. 2015;517(7534):311-320.

14. Kearney CJ, Martin SJ. An inflammatory perspective on necroptosis. Mol Cell. 2017;65(6):965-973.

15. Chan FK, Luz NF, Moriwaki K. Programmed necrosis in the cross talk of cell death and inflammation. Annu Rev Immunol. 2015;33:79-106.

16. Cho YS, et al. Phosphorylation-driven assembly of the RIP1-RIP3 complex regulates programmed necrosis and virus-induced inflammation. Cell. 2009;137(6):1112-1123.

17. He S, et al. Receptor interacting protein kinase-3 determines cellular necrotic response to TNF- $\alpha$. Cell. 2009;137(6):1100-1111.

18. Sun L, et al. Mixed lineage kinase domain-like protein mediates necrosis signaling downstream of RIP3 kinase. Cell. 2012;148(1-2):213-227.

19. Günther C, et al. Caspase- 8 regulates TNF- $\alpha$ induced epithelial necroptosis and terminal ileitis. Nature. 2011;477(7364):335-339.

20. Kaiser WJ, et al. RIP3 mediates the embryonic lethality of caspase-8-deficient mice. Nature. 2011;471(7338):368-372.

21 . Oberst A, et al. Catalytic activity of the caspase8-FLIP(L) complex inhibits RIPK3-dependent necrosis. Nature. 2011;471(7338):363-367.

22. Wittkopf N, et al. Cellular FLICE-like inhibitory protein secures intestinal epithelial cell survival and immune homeostasis by regulating caspase-8. Gastroenterology. 2013;145(6):1369-1379.

23. Zhao Q, et al. RIPK3 mediates necroptosis during embryonic development and postnatal inflammation in Fadd-deficient mice. Cell Rep. 2017;19(4):798-808

24. Lafont E, et al. TBK1 and IKKE prevent TNFinduced cell death by RIPK1 phosphorylation. Nat Cell Biol. 2018;20(12):1389-1399.

25. Xu D, et al. TBK1 suppresses RIPK1-driven apoptosis and inflammation during development and in aging. Cell. 2018;174(6):1477-1491.e19.

26. Geng J, et al. Regulation of RIPK1 activation by TAK1-mediated phosphorylation dictates apoptosis and necroptosis. Nat Commun. 2017;8(1):359.

27. Seo J, et al. CHIP controls necroptosis through ubiquitylation- and lysosomedependent degradation of RIPK3. Nat Cell Biol. 2016;18(3):291-302.

28. Welz PS, et al. FADD prevents RIP3-mediated epithelial cell necrosis and chronic intestinal inflammation. Nature. 2011;477(7364):330-334.

29. Pierdomenico M, et al. Necroptosis is active in children with inflammatory bowel disease and contributes to heighten intestinal inflammation. Am J Gastroenterol. 2014;109(2):279-287.

30. Moriwaki K, Balaji S, McQuade T, Malhotra N, Kang J, Chan FK. The necroptosis adaptor RIPK3 promotes injury-induced cytokine expression and tissue repair. Immunity. 2014;41(4):567-578.

31. Moriwaki K, Balaji S, Bertin J, Gough PJ, Chan FK. Distinct kinase-independent role of RIPK3 in CD11 $\mathrm{c}^{+}$mononuclear phagocytes in cytokine-induced tissue repair. Cell Rep. 2017;18(10):2441-2451.
32. Efeyan A, Comb WC, Sabatini DM. Nutrientsensing mechanisms and pathways. Nature. 2015;517(7534):302-310.

33. Newton K, et al. RIPK3 deficiency or catalytically inactive RIPK1 provides greater benefit than MLKL deficiency in mouse models of inflammation and tissue injury. Cell Death Differ. 2016;23(9):1565-1576

34. Moriwaki K, Balaji S, Chan FK. Border security: the role of RIPK3 in epithelium homeostasis. Front Cell Dev Biol. 2016;4:70.

35. Liu C, Chapman NM, Karmaus PW, Zeng H, Chi H. mTOR and metabolic regulation of conventional and regulatory T cells. J Leukoc Biol. 2015;97(5):837-847.

36. Saxton RA, Sabatini DM. mTOR signaling in growth, metabolism, and disease. Cell. 2017;168(6):960-976

37. Weichhart T, Hengstschläger M, Linke M. Regulation of innate immune cell function by mTOR. Nat Rev Immunol. 2015;15(10):599-614.

38. Dondelinger Y, et al. MK2 phosphorylation of RIPK1 regulates TNF-mediated cell death. Nat Cell Biol. 2017;19(10):1237-1247.

39. He L, et al. mTOR regulates TLR-induced c-fos and Th1 responses to HBV and HCV vaccines. Virol Sin. 2015;30(3):174-189.

40. Kim YC, Guan KL. mTOR: a pharmacologic target for autophagy regulation. JClin Invest. 2015;125(1):25-32.

41. Yilmaz ÖH, et al. mTORC1 in the Paneth cell niche couples intestinal stem-cell function to calorie intake. Nature. 2012;486(7404):490-495.

42. Barron L, Sun RC, Aladegbami B, Erwin CR, Warner BW, Guo J. Intestinal epithelial-specific mTORC1 activation enhances intestinal adaptation after small bowel resection. Cell Mol Gastroenterol Hepatol. 2017;3(2):231-244.

43. Faller WJ, et al. mTORC1-mediated translational elongation limits intestinal tumour initiation and growth. Nature. 2015;517(7535):497-500.

44. Sampson LL, Davis AK, Grogg MW, Zheng Y. mTOR disruption causes intestinal epithelial cell defects and intestinal atrophy postinjury in mice. FASEB J. 2016;30(3):1263-1275.

45. Karki R, et al. NLRC3 is an inhibitory sensor of PI3K-mTOR pathways in cancer. Nature. 2016;540(7634):583-587.

46. Buck MD, Sowell RT, Kaech SM, Pearce EL. Metabolic instruction of immunity. Cell. 2017;169(4):570-586

47. Rooks MG, Garrett WS. Gut microbiota, metabolites and host immunity. Nat Rev Immunol. 2016;16(6):341-352.

48. Inoki K, et al. TSC2 integrates Wnt and energy signals via a coordinated phosphorylation by AMPK and GSK3 to regulate cell growth. Cell. 2006;126(5):955-968.

49. Zhou J, et al. GSK-3 $\alpha$ is a central regulator of age-related pathologies in mice. J Clin Invest. 2013;123(4):1821-1832.

50. Manning BD, Tee AR, Logsdon MN, Blenis J, Cantley LC. Identification of the tuberous sclerosis complex-2 tumor suppressor gene product tuberin as a target of the phosphoinositide 3-kinase/akt pathway. Mol Cell.2002;10(1):151-162.

51. Günther C, et al. Caspase-8 controls the gut response to microbial challenges by $\operatorname{Tnf}-\alpha$ - dependent and independent pathways. Gut. 2015;64(4):601-610.

52. Zhang X, et al. MLKL and FADD are critical for suppressing progressive lymphoproliferative disease and activating the NLRP3 inflammasome. Cell Rep. 2016;16(12):3247-3259.

53. Gruber L, et al. High fat diet accelerates pathogenesis of murine Crohn's disease-like ileitis independently of obesity. PLoS One 2013;8(8):e71661.

54. Kim KA, Gu W, Lee IA, Joh EH, Kim DH. High fat diet-induced gut microbiota exacerbates inflammation and obesity in mice via the TLR4 signaling pathway. PLoS One. 2012;7(10):e47713

55. Hu S, et al. mTOR inhibition attenuates dextran sulfate sodium-induced colitis by suppressing T cell proliferation and balancing TH1/TH17/ Treg profile. PLoS One. 2016;11(4):e0154564.

56. Nagahama Y, et al. Regnase-1 controls colon epithelial regeneration via regulation of $\mathrm{mTOR}$ and purine metabolism. Proc Natl Acad Sci U S A 2018;115(43):11036-11041.

57. Jaco I, et al. MK2 phosphorylates RIPK1 to prevent TNF-induced cell death. Mol Cell. 2017;66(5):698-710.e5

58. Kimura T, Jain A, Choi SW, Mandell MA, Johansen T, Deretic V. TRIM-directed selective autophagy regulates immune activation. Autophagy. 2017;13(5):989-990.

59. Liu T, et al. TRIM11 Suppresses AIM2 inflammasome by degrading AIM2 via p62-dependent selective autophagy. Cell Rep. 2016;16(7):1988-2002.

60. Ronan B, et al. A highly potent and selective Vps34 inhibitor alters vesicle trafficking and autophagy. Nat Chem Biol. 2014;10(12):1013-1019.

61. Xiao H, et al. The Toll-interleukin-1 receptor member SIGIRR regulates colonic epithelial homeostasis, inflammation, and tumorigenesis. Immunity. 2007;26(4):461-475.

62. Koo GB, et al. Methylation-dependent loss of RIP3 expression in cancer represses programmed necrosis in response to chemotherapeutics. Cell Res. 2015;25(6):707-725.

63. Bozec D, Iuga AC, Roda G, Dahan S, Yeretssian G. Critical function of the necroptosis adaptor RIPK3 in protecting from intestinal tumorigenesis. Oncotarget. 2016;7(29):46384-46400.

64. Xiao H, et al. Loss of single immunoglobulin interlukin-1 receptor-related molecule leads to enhanced colonic polyposis in Apc(min) mice. Gastroenterology. 2010;139(2):574-585.

65. Moriwaki K, Chan FK. Regulation of RIPK3and RHIM-dependent necroptosis by the proteasome. J Biol Chem. 2016;291(11):5948-5959.

66. Chen L, Brewer MD, Guo L, Wang R, Jiang P, Yang X. Enhanced degradation of misfolded proteins promotes tumorigenesis. Cell Rep. 2017;18(13):3143-3154.

67. Matsuzawa-Ishimoto Y, et al. Autophagy protein ATG16L1 prevents necroptosis in the intestinal epithelium. JExp Med. 2017;214(12):3687-3705

68. Liu ZY, et al. Necrostatin-1 reduces intestinal inflammation and colitis-associated tumorigenesis in mice. Am J Cancer Res. 2015;5(10):3174-3185.

69. Vlantis K, et al. NEMO prevents RIP Kinase 1-mediated epithelial cell death and chronic 
intestinal inflammation by NF- $\mathrm{KB}$-dependent and -independent functions. Immunity. 2016;44(3):553-567.

70. Seifert L, et al. The necrosome promotes pancreatic oncogenesis via CXCL1 and Mincle-induced immune suppression. Nature. 2016;532(7598):245-249.

71. Seehawer M, et al. Necroptosis microenvironment directs lineage commitment in liver cancer. Nature. 2018;562(7725):69-75.

72. Morgan MJ, Kim YS. The serine threonine kinase RIP3: lost and found. BMB Rep. 2015;48(6):303-312.

73. Moriwaki K, Bertin J, Gough PJ, Orlowski GM, Chan FK. Differential roles of RIPK1 and RIPK3 in TNF-induced necroptosis and chemotherapeutic agent-induced cell death. Cell Death Dis. 2015;6:e1636.

74. He GW, et al. Regression of apoptosis-resistant colorectal tumors by induction of necroptosis in mice. J Exp Med. 2017;214(6):1655-1662.

75. Brumatti $G$, et al. The caspase- 8 inhibitor emricasan combines with the SMAC mimetic birinapant to induce necroptosis and treat acute myeloid leukemia. Sci Transl Med. 2016;8(339):339ra69.

76. Kwiatkowski DJ, et al. A mouse model of TSC1 reveals sex-dependent lethality from liver hemangiomas, and up-regulation of p70s6 kinase activity in Tsc1 null cells. Hum Mol Genet.
2002;11(5):525-534.

77. Gangloff YG, et al. Disruption of the mouse mTOR gene leads to early postimplantation lethality and prohibits embryonic stem cell development. Mol Cell Biol. 2004;24(21):9508-9516.

78. Madison BB, Dunbar L, Qiao XT, Braunstein $\mathrm{K}$, Braunstein E, Gumucio DL. Cis elements of the villin gene control expression in restricted domains of the vertical (crypt) and horizontal (duodenum, cecum) axes of the intestine. J Biol Chem. 2002;277(36):33275-33283.

79. Vereecke L, et al. Enterocyte-specific A20 deficiency sensitizes to tumor necrosis factorinduced toxicity and experimental colitis. JExp Med. 2010;207(7):1513-1523. 